\title{
Evolutionary relationships between the varieties of green algae Pediastrum boryanum and $P$. duplex s.l. (Chlorophyceae, Hydrodictyaceae)
}

\author{
Joanna LENARCZYK* \& Marta SAŁUGA \\ W. Szafer Institute of Botany, Polish Academy of Sciences, Lubicz 46, 31-512 Kraków, Poland; *Corresponding \\ authore-mail: j.lenarczyk@botany.pl
}

\begin{abstract}
The infraspecific relationships of the two most variable species, Pediastrum boryanum and P. duplex s.l. identified on the basis of morphological criteria, have been poorly studied so far. The present study focused on 12 original strains isolated from Polish water bodies and 29 strains from the GenBank database, which represent $P$. boryanum, $P$. duplex s.l. varieties and other morphologically similar taxa, against the background of 14 other strains of the family Hydrodictyaceae. In order to estimate the level of congruence between the phylogeny and the classical taxonomic system based on morphological criteria, the strains of $P$. boryanum and P. duplex s.l., and other morphologically similar taxa were subjected to parallel phylogenetic analyses applying Bayesian and Maximum Likelihood methods to combined molecular data (26S rDNA and $r b c \mathrm{~L}$ cpDNA) and morphological analyses based on light and scanning electron microscopy, and iconographic documentation of almost all strains published elsewhere. The gene topologies revealed many discrepancies in the morphological features used to delimit the analysed taxa. The polyphyly of both $P$. boryanum and $P$. duplex s.l. was confirmed. Pseudopediastrum boryanum var. cornutum (formerly P. boryanum var. cornutum) formed a well supported monophyletic clade, whereas other varieties, including var. boryanum, brevicorne and longicorne, proved to be complex taxa. Previous description of Lacunastrum gracillimum (formerly P. duplex var. gracillimum) was well supported. A clade composed mostly of European strains identified as P. duplex var. rugulosum should be regarded as this taxon, whereas clades containing strains exhibiting $P$. duplex var. rugulosum-like morphology represent new taxa. Taxonomical changes within both $P$. boryanum and $P$. duplex s.l. are required on the basis of a higher number of strains and detailed morphometric data.
\end{abstract}

Key words: Bayesian phylogenetic analysis, Hydrodictyaceae, Maximum Likelihood, morphological variability, Pediastrum boryanum, Pediastrum duplex, Pseudopediastrum, 26S rDNA, rbcL cpDNA

\section{INTRODUCTION}

Pediastrum boryanum and $P$. duplex s.1. are microscopic green algal species of the family Hydrodictyaceae, order Sphaeropleales, class Chlorophyceae, which occur commonly worldwide in the phytoplankton, especially of fresh and coastal waters with higher conductivity (KOMÁREK \& JANKOVSKÁ 2001; LENARCZYK 2015). Both were described in the first half of the nineteenth century: P. boryanum by MeNeGHINI (1840), who emended its original description as Helierella boryana (TURPIN 1828), and $P$. duplex by MeYen (1829). As with other representatives of the genus Pediastrum Meyen, the species form flat, star-shaped colonies of cells, called coenobia. As a result of mitotic divisions of a single maternal cell, the cells in a coenobium are all of the same age. The number of cells, usually 8,16 or 32 , is fixed $\left(2^{n}\right)$ and does not change during coenobium growth (КомÁREK \& FotT 1983; LeNARCZYK 2016).
In the most recent key to identification of Pediastrum taxa by KoMÁREK \& JANKovsKá (2001), nine varieties of $P$. boryanum and four varieties of $P$. duplex are distinguished. What is typical of $P$. boryanum coenobia is a lack of perforations between the U-shaped marginal cells, having two lobes ending with hyaline processes of various length, and the polygonal inner cells, as well as a reticulate wall ornamentation composed of granules situated regularly in the corners of a triangular mesh. In contrast, $P$. duplex always forms perforated coenobia with slender or robust $\mathrm{H}$-shaped cells, its marginal cells have two lobes which end with very short hyaline processes and their wall surface is smooth (without any wall ornamentation), wrinkled or reticulate with irregularly situated granules (PARRA 1979; KOMÁREK \& JANKOVSKÁ 2001; LENARCZYK 2014; LENARCZYK \& WOŁOWSKI 2016).

According to KoMÁREK \& JANKOVSKÁ (2001), both species belong to the most morphologically variable 
Pediastrum taxa. The variability does not only follow a genetic basis, but also results from ontogenetic changes the position of cells in the coenobium - as well as plasticity under different growth environment conditions, such as salinity, composition and concentration of nutrients, and light intensity (CHANG \& CHANG-SCHNEIDER 1980; NeUSTUPA \& HodAČ 2005; Rojo et al. 2007; LENARCZYK 2014, 2016; LENARCZYK \& MCMANus 2016; SuTKOWY 2017). As a consequence of the great variability, several infraspecific taxonomic classifications of $P$. boryanum and $P$. duplex have been introduced, putting different emphasis on individual morphological features (e.g. RACIBORSKi 1889; SuleK 1969; PARRA 1979). These changes in the systematics have resulted in publication of a great number of taxon names, most of them currently regarded as synonyms (PARRA 1979).

In recent years, the phylogenetic studies by McManus \& Lewis (2005, 2011), BuchHeIm et al. (2005), JENA et al. (2014) and LENARCZYK \& McMAnus (2016) have thrown little light on the systematics of $P$. boryanum and P. duplex s.l.

MCMANUS \& LEWIS (2005) analysed 26S, 5.8S and ITS -2 rDNA of single strains identified as $P$. boryanum varieties, including the vars. brevicorne A. Braun, cornutum Sulek and forcipatum Parra, two other strains without varietal status, plus eight strains of $P$. duplex, including only one identified to variety level (asperum (A. Braun) Hansgirg).

BuCHHEIM et al. (2005) published their results on phylogenetic relationships within the genus Pediastrum based on 18S, 26S and ITS-2 rDNA regions, analysing only two strains of $P$. boryanum, including one identified as the var. cornutum, and two strains of $P$. duplex. They split the genus Pediastrum s.l. into five genera, renaming $P$. boryanum as Pseudopediastrum boryanum (Turpin) E. Hegewald.

Later, MCMANUS \& LEWIS (2011) analysed nuclear (26S rDNA) and chloroplast ( $r b c \mathrm{~L} c p D N A)$ sequences of Ps. boryanum and P. duplex. Among the Ps. boryanum strains were two identified as the var. cornutum forming a common clade, three strains identified as the var. brevicorne belonging to separate clades, single strains identified as the var. boryanum and var. forcipatum related to strains of Ps. boryanum or an unidentified Pseudopediastrum sp. Ps. boryanum was shown to be paraphyletic, with Ps. kawraiskyi (Schmidle) E. Hegewald and P. integrum Nägeli being nested within a clade composed of most PS. boryanum strains, except for one strain identified as Ps. boryanum var. brevicorne. Similarly, analysing 36 strains of $P$. duplex, four were identified as the var. gracillimum W. West et G.S. West and single strains as vars. duplex, rugulosum Raciborski and asperum, with $P$. duplex being considered nonmonophyletic. Some strains identified as $P$. duplex were more closely related to $P$. angulosum (Ehrenberg) ex Meneghini or $P$. cf. alternans NyGAARD than to other $P$. duplex strains. McManus \& LEWIS (2011) pointed out that the presence of intercellular perforations and the type of cell wall surface do not generally correspond with the phylogeny of $P$. duplex. However, the phylogenetic studies on $P$. duplex, supported by precise morphological analyses based on geometric morphometric methods, gave the opportunity to transfer the variety gracillimum to a new genus Lacunastrum, as L. gracillimum (W. West et G. S. West) H. McManus (McManus et al. 2011).

In the next studies on Pediastrum phylogeny, JENA et al. (2014) sequenced 18S and ITS rDNA regions of five $P$ s. boryanum, including four which were identified as Ps. boryanum and one as Ps. brevicorne (A. Braun) Jena et C. Bock (formerly P. boryanum var. brevicorne), forming separate evolutional lineages, as well as seven strains of $P$. duplex and two Lacunastrum gracillimum. A single strain having $\mathrm{H}$-shaped cells typical of $P$. $d u$ plex, but with marginal cells situated somewhat out of the plane of the coenobium, was described as Sorastrum pediastriforme Jena et $\mathrm{C}$. Bock on the basis of its close relationship with a few strains of the three-dimensional genus Sorastrum Kützing.

In the most recent paper on Pediastrum phylogeny, LENARCZYK \& MCMANUs (2016) used phylogenetic and geometric morphometric methods in order to test taxonomical boundaries of $P$. alternans Nygaard. They determined the phylogenetic position, based on three molecular markers (26S, ITS and $r b c \mathrm{~L})$, of two strains identified as Ps. alternans and one as Ps. boryanum var. longicorne among several other representatives of the family Hydrodictyaceae published elsewhere (McManus \& LEWIS 2005, 2011).

In all these phylogenetic studies, a low number of strains identified as $P$. boryanum and $P$. duplex varieties were included. The studies did not allow reliable conclusions on phylogenetic relationships of most $P$. boryanum and $P$. duplex varieties and the evolution of their morphology. Consequently, the present study focused on a rich collection of original and published DNA sequences of monoclonal strains identified as $P$. boryanum and $P$. duplex varieties, as well as those with morphology typical of, or close to, the morphology of these species. Detailed morphological analysis consisted in identification of the size of coenobia (number of cells) and perforations between cells, the marginal cell shape and type of the cell wall surface. Phylogenetic analysis was based on two molecular markers (26S nuclear DNA and $r b c \mathrm{~L}$ chloroplast DNA) used jointly. The aim of the present study was to estimate the level of congruence between the phylogeny of $P$. boryanum, $P$. duplex s.l., other similar species and the classical taxonomical system based on morphological criteria.

\section{Materials AND Methods}

A total of fifty five Hydrodictyaceae strains originating from various ecosystems all over the world (Europe - 34 strains, North America -8 strains, Australia -6 strains, Africa -2 
strains, and Asia - 1 strain; 4 strains without known locality) were subjected to morphological and/or phylogenetic analysis (Figs 1-6, Tables 1, S1). Among them were forty one strains representing $P$. boryanum, $P$. duplex s.l. varieties and other morphologically similar taxa, as well as fourteen strains representing other taxa of the family Hydrodictyaceae, including Hydrodictyon spp., Monactinus spp., Pediastrum angulosum, P. braunii, Parapediastrum biradiatum, Pseudopediastrum kawraiskyi, Sorastrum spp. and Stauridium tetras.

Morphological analysis. Altogether, thirty nine strains exhibiting morphology typical of, or close to, Pediastrum boryanum s.l. and $P$. duplex s.l. were subjected to morphological analysis (Table 1). Two strains, ACOI 893 and ACOI 1009 included in the phylogenetic analysis (Fig. 1) represented the varieties of the species of interest (P. duplex var. duplex and Pseudopediastrum boryanum var. cornutum, respectively), but their morphology could not be analysed because of the lack of iconographic documentation in the literature.

Among thirty nine strains subjected to morphological analysis were fifteen strains examined by the authors using light and scanning electron microscopes, twenty strains examined based on microphotographs and drawings published elsewhere (PARra 1979; Nielsen 2000; BuchHeim et al. 2005; McManus \& Lewis 2005, 2011; McManus et al. 2011; Jena et al. 2014; the website of the Algae Culture Collection COIMBRA: http:// acoi.ci.uc.pt/), as well as four strains examined both on the basis of our own observations and iconographic documentation in the above mentioned literature (Table 1).

Among the strains subjected to our own microscopic observations were twelve original monoclonal strains isolated from field samples collected from lakes and ponds in Poland and seven strains acquired from European algae culture collections: three from the Culture Collection of Algae at Göttingen University in Germany (SAG 29.81, SAG 87.81, SAG 2440), two from the Algae Culture Collection COIMBRA in Portugal (ACOI 75, ACOI 685) and two from the algae culture collection of the W. Szafer Institute of Botany, Polish Academy of Sciences in Poland (01.021213, 04.080210).

The original strains were started with single coenobia, which were isolated using a Narishige MM 188 micromanipulator (Tokyo, Japan) and Jenaval microscope (Carl Zeiss, Jena, Germany). The strains were maintained in sterile liquid $\mathrm{L}-\mathrm{S}_{2} \mathrm{~T}_{2}$ medium (LENARCZYK 2014) at ca $22^{\circ} \mathrm{C}$ in a Bolarus G-18-2 (Bochnia, Poland) phytotron or in a sterile liquid modified Waris-H medium (LENARCZYK \& McManus 2016) at ca $20^{\circ} \mathrm{C}$, 15-20 $\mu \mathrm{mol}$ photons. $\mathrm{m}^{-2} \cdot \mathrm{sec}^{-1}$, a 12:12 light:dark regime in a POL-EKO-APARATURA ST2 COMF/S (Wodzisław Śląski, Poland) phytotron (Table 1). The strains were deposited in the algae culture collection of the W. Szafer Institute of Botany PAS (Kraków, Poland). Strains acquired from European algae culture collections were kept in a modified Waris-H medium under the same culture conditions.

Light microscopy (LM) observations were made using a Nikon DS-Fil (Tokyo, Japan) digital camera and Nikon Optiphot-2 (strains cultured in L- $\mathrm{S}_{2} \mathrm{~T}_{2}$ medium) or Nikon Eclipse $\mathrm{N} i$ (strains cultured in a modified Waris-H medium) microscopes (Tokyo, Japan). For scanning electron microscopy (SEM), the samples were prepared and analysed as presented in LENARCZYK \& MCMANUs (2016).

The strains were morphologically compared paying attention to the most prominent morphological features. These were the (1) size of coenobia, (2) size of perforations in coenobia, (3) marginal cell shape and (4) cell wall surface (Table 1). The size of coenobia was described as small for strains with up to 32-celled coenobia or as large for strains with up to 128-celled coenobia. The analysed coenobia had small, large or no perforations. To minimize the error in defining shape of the marginal cells resulting from morphological variability and the limited source of available microphotographs, only five general types of marginal cell shape were distinguished: rounded or long U-shape, robust or slender H-shape, and rounded $\mathrm{O}-$-shape. The cell wall surface was described as smooth or possessing various types of ornamentation, including wrinkles, ridges, triangular mesh, polygonal mesh, thickenings and granules which were situated loosely, of medium density or as dense on the surface. The nomenclature of the surface ornamentation follows LENARCZYK (2014). The names of the published strains were kept as given by previous authors, whereas the names of original strains were given on the basis of morphological observations and identification according to the taxonomic system of KOMÁREK \& JANKOVSKÁ (2001).

Phylogenetic analysis. Two molecular markers, nuclear LSU (26S rDNA) and chloroplast RUBISCO LSU $(r b c \mathrm{~L})$, were sequenced for each of the twelve original strains originating from Polish water bodies. Both gene regions of fourty three other strains of the family Hydrodictyaceae were obtained from the GenBank database (National Centre for Biotechnology Information [NCBI] http:/www.ncbi.nlm.nih.gov/). These strains were published elsewhere by BuchHEIM et al. (2005), MCMANus \& Lewis (2005, 2011), JenA et al. (2014) and LeNARCZYK \& McManus (2016) (Table S1).

DNA extraction, amplification and sequencing protocols of the twelve original Polish strains follow LENARCZYK \& McManus (2016). Primers for amplification and sequencing of the 26S rDNA and $r b c \mathrm{~L}$ cpDNA regions are listed in Table 2 . The PCR conditions for the $26 \mathrm{~S}$ rDNA reactions included $94{ }^{\circ} \mathrm{C}$ for $3 \mathrm{~min}$ and then 35 cycles of $94{ }^{\circ} \mathrm{C}$ for $1 \mathrm{~min}, 59$ ${ }^{\circ} \mathrm{C}$ for $1 \mathrm{~min}$ and elongation at $72{ }^{\circ} \mathrm{C}$ for $1 \mathrm{~min}$, with a final extension step at $72{ }^{\circ} \mathrm{C}$ for $10 \mathrm{~min}$. The PCR conditions for the $r b c \mathrm{~L}$ cpDNA reactions were $94{ }^{\circ} \mathrm{C}$ for $3 \mathrm{~min}$ and then 30 cycles of $94{ }^{\circ} \mathrm{C}$ for $1 \min 15 \mathrm{sec}, 56^{\circ} \mathrm{C}$ for $2 \mathrm{~min}$ and elongation at $72{ }^{\circ} \mathrm{C}$ for $2 \mathrm{~min} 15 \mathrm{sec}$, with a final extension step at $72{ }^{\circ} \mathrm{C}$ for $7 \mathrm{~min}$.

The consensus 26S and $r b c \mathrm{~L}$ sequences of the original strains (Tables $1 \& \mathrm{~S} 1$ ) were automatically built with Variant Reporter, ver. 1.0 (Applied Biosystems). Phylogenetic analyses were performed jointly on the $26 \mathrm{~S}$ and $r b c \mathrm{~L}$ sequences. Neochloris aquatica Starr, from the closely related family Neochloridaceae, was selected as the outgroup. Examined sequences were edited using Geneious, ver. 10.1.3 (Biomatters, USA), and aligned with the Geneious alignment with Muscle algorithm set to default parameters. Regions of partially incomplete data at the beginning and end of the sequences were identified and manually excluded from subsequent analyses with BioEdit, ver.7.2.5 (HaLl 1999). In case of the 26S alignment, 416 bp long fragment of Parapediastrum biradiatum (UTEX 37) was excluded from subsequent analyses because of insertion and deletion (indel) polymorphism. Similarly, two substantial indels, 1782 and 1696 bp long fragments, were eliminated from the middle part of the $r b c \mathrm{~L}$ sequence alignment. Here, we identified indel polymorphism for six different accessions: Lacunastrum gracillimum (Hg2004-1), L. gracillimum (LN0201NC), L. gracillimum (SML0401VA), Pediastrum duplex var. duplex (ACOI 893), Pseudopediastrum boryanum var. cornutum (UTEX LB470) and Ps. kawraiskyi (SAG 35.81). Consequently, alignments of $26 \mathrm{~S}$ and $r b c \mathrm{~L}$ sequences were respectively 1940 and $1229 \mathrm{bp}$ long. The aligned sequences were concatenated into a single data matrix (26S 


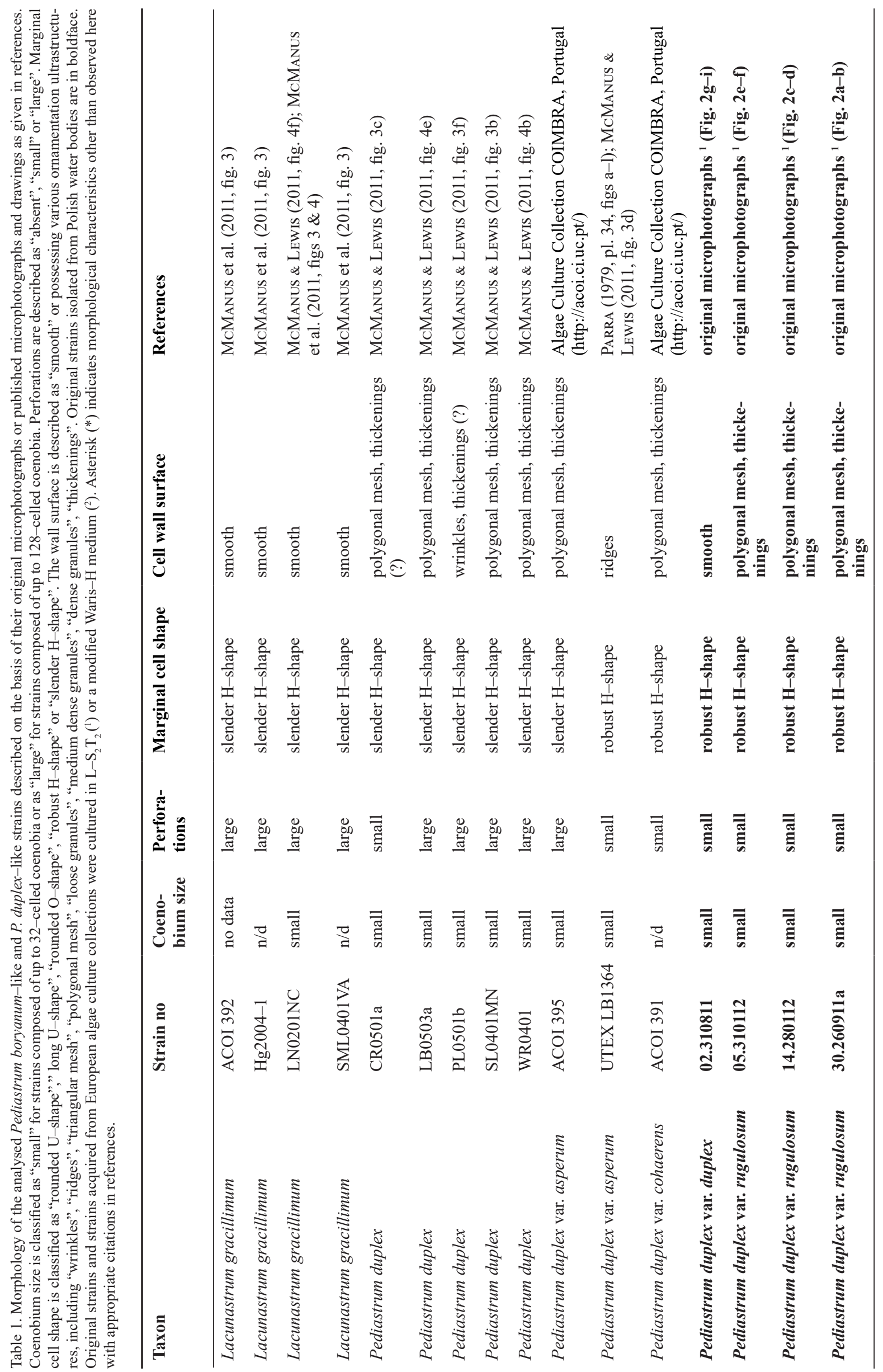




\begin{tabular}{|c|c|c|c|c|c|c|c|c|c|c|c|c|c|}
\hline 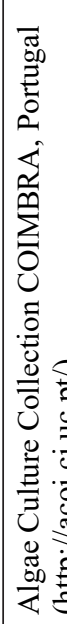 & 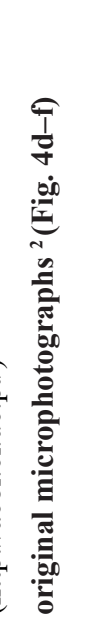 & 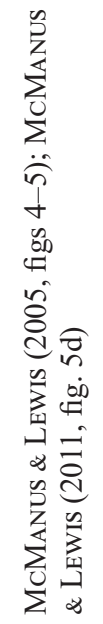 & 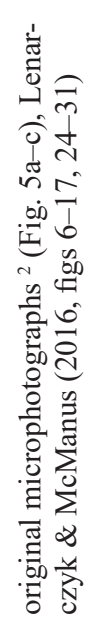 & 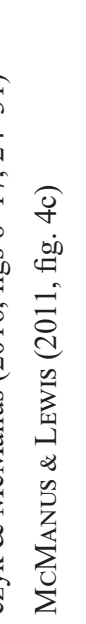 & 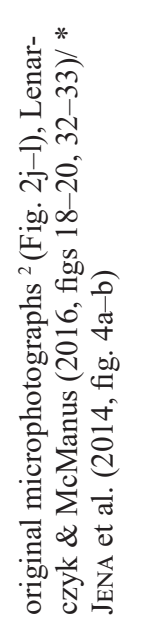 & 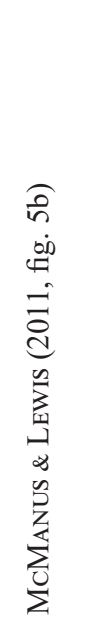 & 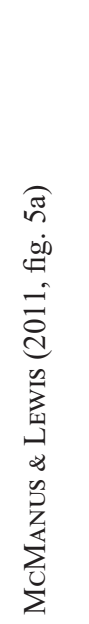 & 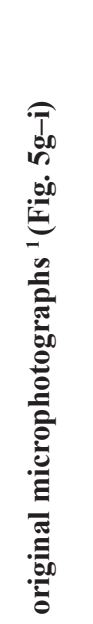 & 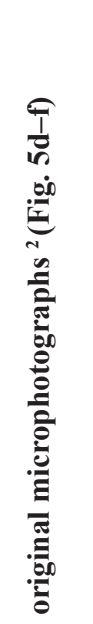 & 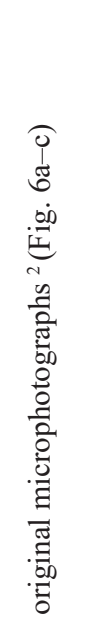 & 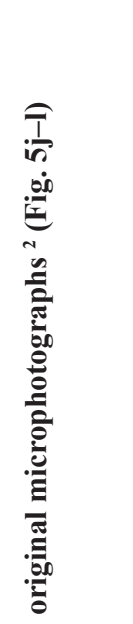 & 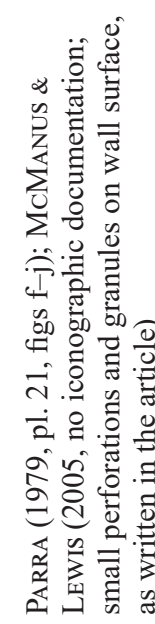 & 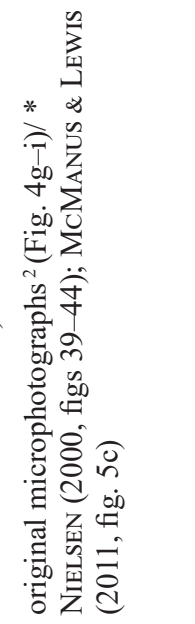 \\
\hline Te & 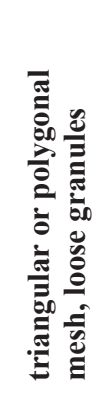 & 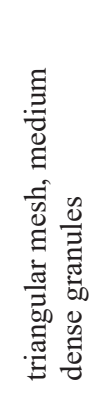 & 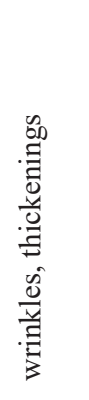 & $\frac{\frac{y_{3}^{3}}{3}}{\frac{1}{3}}$ & 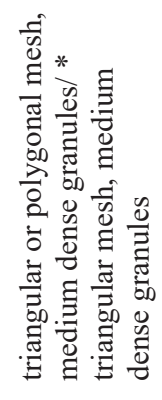 & 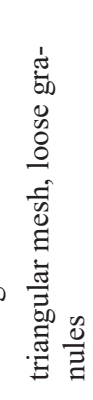 & 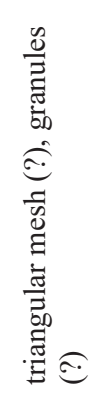 & 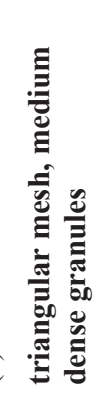 & 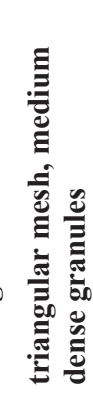 & 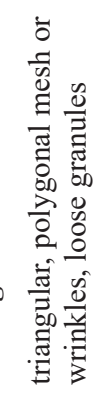 & 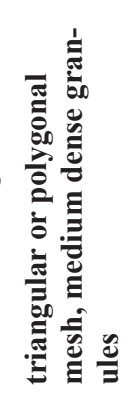 & 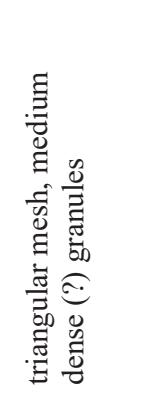 & 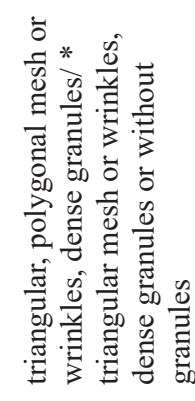 \\
\hline 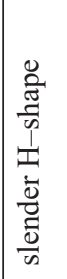 & 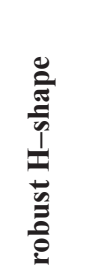 & 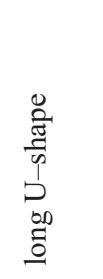 & 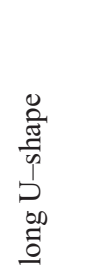 & 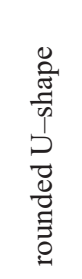 & 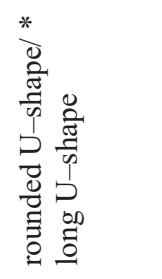 & 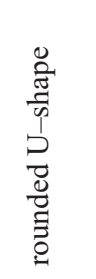 & 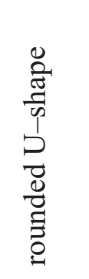 & 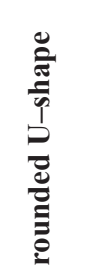 & 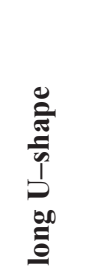 & 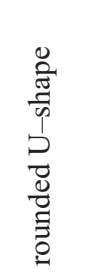 & 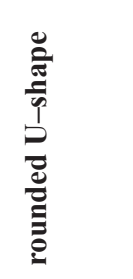 & 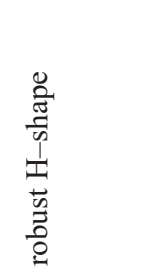 & 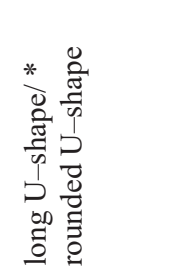 \\
\hline 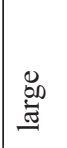 & $\underset{\Xi}{\mathbb{E}}$ & $\begin{array}{l}\overrightarrow{\bar{J}} \\
\overrightarrow{0} \\
\vec{\omega}\end{array}$ & 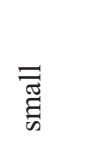 & $\begin{array}{l}\overline{\bar{\Xi}} \\
\text { है }\end{array}$ & 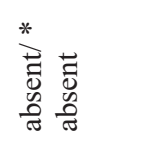 & 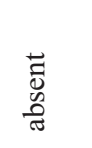 & $\begin{array}{l}\overrightarrow{\tilde{J}^{\prime}} \\
\text { 岕 }\end{array}$ & 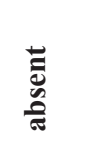 & 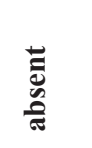 & 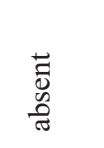 & $\overline{\overline{\bar{W}}}$ & 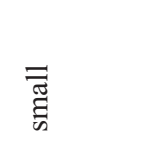 & 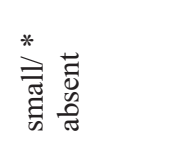 \\
\hline 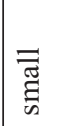 & 驫 & 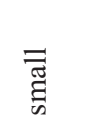 & 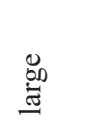 & $\begin{array}{l}\overline{\bar{\Xi}} \\
\text { ज्ञ }\end{array}$ & 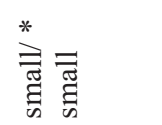 & 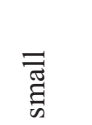 & 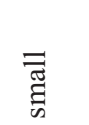 & $\stackrel{8}{0}$ & $\overline{\overline{\bar{J}}}$ & 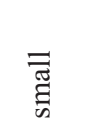 & $\overline{\overline{\tilde{T}}}$ & $\overline{\bar{\sigma}}$ & $\begin{array}{l}\text { 总 } \\
\text { 总 }\end{array}$ \\
\hline 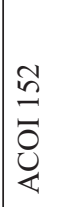 & 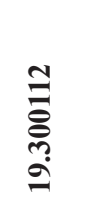 & 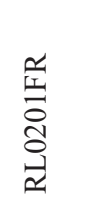 & $\begin{array}{l}\stackrel{M}{\vec{I}} \\
\stackrel{\Delta}{\sigma} \\
\dot{0}\end{array}$ & 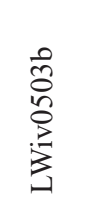 & 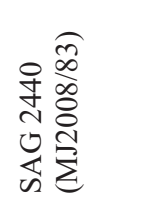 & 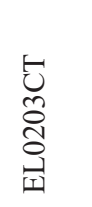 & 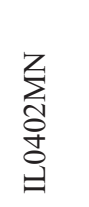 & 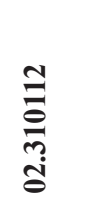 & 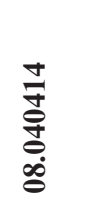 & 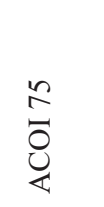 & 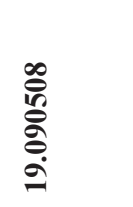 & 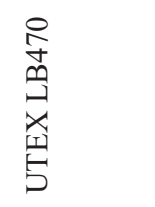 & \begin{tabular}{l}
$\vec{\infty}$ \\
$\infty$ \\
$\infty$ \\
0 \\
\multirow{\omega}{\infty}{}
\end{tabular} \\
\hline 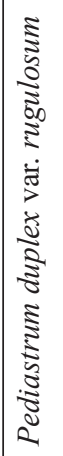 & 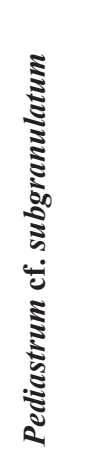 & 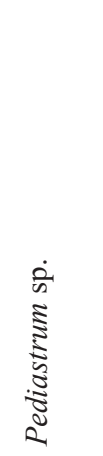 & 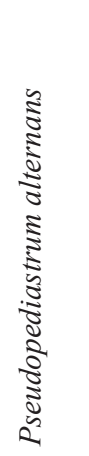 & 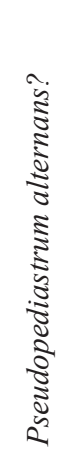 & 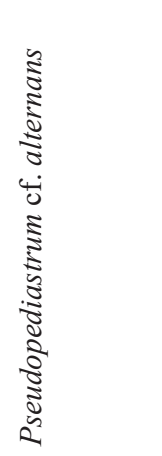 & 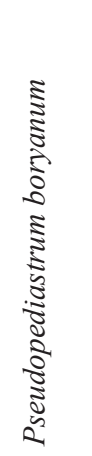 & 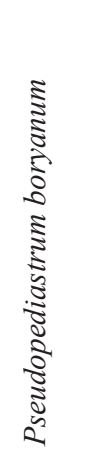 & 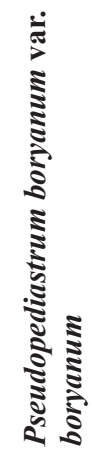 & 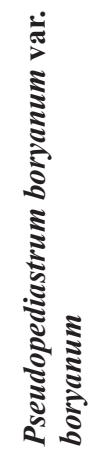 & 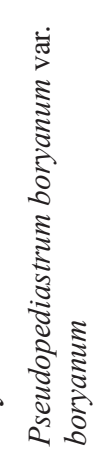 & 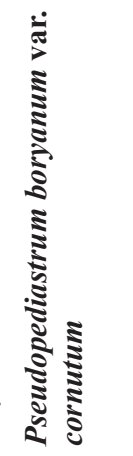 & 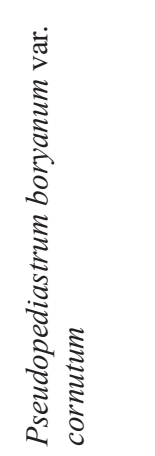 & 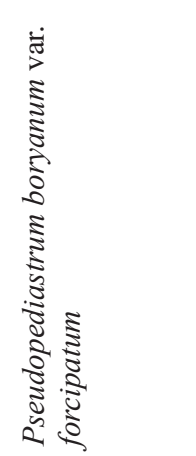 \\
\hline
\end{tabular}




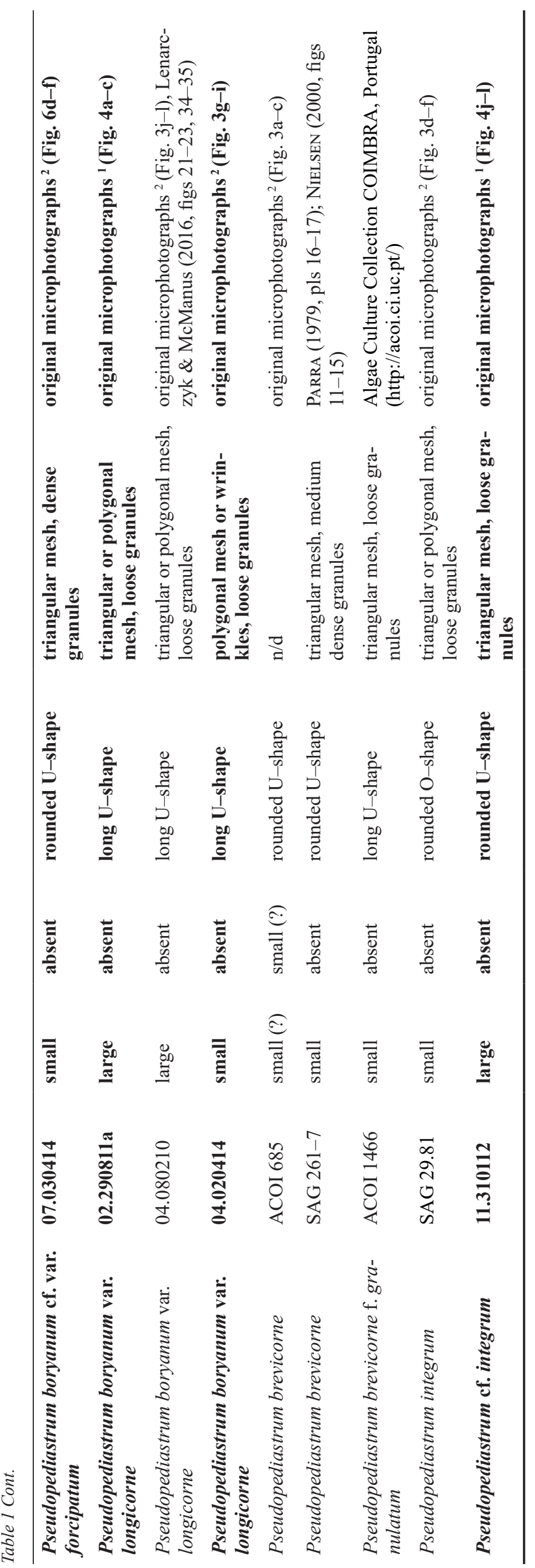

$+r b c \mathrm{~L}$ ) with SeaView, ver. 4.6.1. (Gour et al. 2010). Finally, the concatenated alignment of the $26 \mathrm{~S}$ and $r b c \mathrm{~L}$ dataset consisted of 3169 characters.

Phylogenetic analyses were performed using two methods: Maximum Likelihood (ML) and Bayesian inference (BI). ML was performed in RAxML, ver. 8.2.11 via the Geneious, ver. 10.1.3 (Biomatters, USA) (STAMATAKIS et al. 2008; StAMATAKis 2014). ML branch support was estimated using 1000 rapid ML bootstrap pseudoreplicates. We chose the GTRGAMMA nucleotide model because it includes the parameter $\mathrm{G}$ for rate heterogeneity among sites. In RAxML, by default, $\mathrm{G}$ has 25 rate categories making the estimation of proportion of invariable sites (I) unnecessary because $\mathrm{G}$ mathematically accounts for I (Stamatakis 2006; NGuyen et al. 2015).

Prior to BI analysis, jmodeltest, ver. 2.1.6 (DARRIBA et al. 2012), was used to select a $\operatorname{TrN}+\mathrm{I}+\mathrm{G}$ model for $26 \mathrm{~S}$, and a GTR $+\mathrm{I}+\mathrm{G}$ for $r b c \mathrm{~L}$. Both identified nucleotide evolution models were selected on the basis of Bayesian Information Criterion (Schwarz 1978). BI analysis was performed with MrBayes, ver.3.2.2 (RoNQUIST et al. 2012). The concatenated data matrix was divided into two partitions, each corresponding to a continuous DNA region. The MCMC algorithm was run for $10^{6}$ generations with one cold and three heated chains, and storing one tree every 100 generations. The stationary phase was reached after 847.000 generations. Average standard deviation of split frequencies was 0.009968 . The first $25 \%$ of trees were discarded as burn-in, and a 50\% majority-rule consensus tree was constructed with posterior probabilities (PP) as a measure of clade support.

Consensus topologies of ML and BI trees with maximum likelihood bootstrap percentages and Bayesian branch support values were compiled and drawn using FigTree, ver. 1.4.2 (http://tree.bio.ed.ac.uk/software/figtree/). No topological conflicts were found between ML and BI analyses resulted in identical phylogenetic trees. Thus, we presented one phylogenetic tree (Fig. 1) elaborated using CorelDRAW X4, ver.14 (Corel Corporation).

\section{Results}

A total of 22 strains exhibiting morphology typical of, or close to, Pediastrum boryanum s.1. and 17 strains morphologically typical of, or close to, P. duplex s.1. were subjected to morphological analysis (Table 1). The Pediastrum boryanum-like strains represented 14 taxa, including Pseudopediastrum boryanum (Turpin) E. Hegewald (2 strains; formerly P. boryanum (Turpin) Meneghini), Ps. boryanum var. boryanum (3 strains), Ps. boryanum var. cornutum Sulek (3 strains), Ps. boryanum var. forcipatum Corda (1 strain), Ps. boryanum cf. var. forcipatum (1 strain), Ps. boryanum var. longicorne Reinsch (4 strains, including the synonym Ps. brevicorne (A. Braun) Jena et C. Bock f. granulatum (Raciborski) Parra), Ps. brevicorne (2 strains; formerly P. boryanum var. brevicorne A. Braun), Ps. alternans (Nygaard) Jena et C. Bock (1 strain), Ps. alternans? (1 strain), Ps. cf. alternans (1 strain), Ps. integrum (Nägeli) Jena et C. Bock (1 strain), Ps. cf. integrum (1 strain), 
Table 2. Primers used in amplifying and sequencing the $26 \mathrm{~S}$ rDNA and $r b c \mathrm{~L}$ cpDNA numbered from the 5' end. $\mathrm{F}$ indicates forward and $\mathrm{R}$ indicates reverse primers.

\begin{tabular}{ll}
\hline Primers for 26S rDNA & Sequence (5'-3') \\
\hline ITS4rcR & GCATATCAATAAGCGGAGGA \\
LS1R & GTACCGTGAGGGAAAGA \\
LS13F & GCTTACCAAAAATGGC \\
LS14R & GGCCATTTTTGGTAAGC \\
LS18F & TCCCCTTGTCCGTACCAGT \\
LS20F & TTTCTCCACTTCAGTCTTC \\
26F & CAGAGCACTGGGCAGAAATCAC \\
26BF & GGTCCGTGTTTCAAGACG \\
26BrcR & CCCGTCTTGAAACACGGA \\
Primers for $\boldsymbol{r b c \mathbf { L } \text { cpDNA }}$ & Sequence (5'-3') \\
M35F & GGRWTTAAAGCTGGTGTAAAAGACT \\
M379F & GGTTTCAAAGCTCTTCGTGC \\
M633F & CATGCGTTGGAGAGACCGT \\
M650R & CGGTCTCTCCAACGCATGA \\
M955F & CGTWTATCTGGTGGAGAYC \\
M1010R & CCTTCAAGTTTACCTACAAC \\
M1161R & CATGTGCCATACGTGAATAC \\
M1338R & AATCWGGACTCCATTTACAAGC \\
\hline
\end{tabular}

P. cf. subgranulatum Komárek et Jankovská (1 strain) and Pediastrum sp. (1 strain). They usually formed small coenobia without perforations between rounded U-shaped marginal cells and polygonal inner cells, which were covered with triangular mesh and granulations of various density. Only exceptionally did the strains also have large coenobia composed of more than 32 cells, coenobia with perforations, long U-shaped, rounded O-shaped or even robust $\mathrm{H}$-shaped marginal cells, as well as polygonal mesh, wrinkles and thickenings on the cell wall surface.

In contrast, the $P$. duplex-like strains were represented only by small perforated coenobia, usually with large holes between slender or robust $\mathrm{H}$-shaped cells. They were identified as 6 taxa, including $P$. duplex Meyen (5 strains), $P$. duplex var. duplex (2 strains), $P$. duplex var. asperum (A. Braun) Hansgirg (2 strains), $P$. duplex var. cohaerens Bohlin (1 strain), P. duplex var. rugulosum Raciborski (4 strains) and Lacunastrum gracillimum (W. West et G.S. West) H. McManus (4 strains; formerly $P$. duplex var. gracillimum W. West et G.S. West) (Tables 1, S1).

In the phylogenetic tree from Bayesian analysis of joint 26S rDNA and $r b c \mathrm{~L}$ cpDNA (Fig. 1), Pediastrum boryanum-like and $P$. duplex-like strains, as well as strains representing other morphological types, including those of Hydrodictyon, Monactinus, $P$. angulosum and Ps. kawraiskyi, form a well supported clade (Bayesian Posterior Probability BPP 0.998, Maximum Likelihood ML 66), which is sister to a clade composed of Sorastrum and Parapediastrum strains. The strains of $P$. braunii and Stauridium tetras form outer branches.

\section{Pediastrum duplex-like strains}

All the isolates of Lacunastrum gracillimum from Europe and North America are gathered in a well resolved lineage (GROUP I in Fig. 1; BPP 1, ML 100), which, together with two isolates of Monactinus spp. gathered in a sister lineage, form a well supported branch (BPP 0.987, ML 56). The L. gracillimum strains share similar morphological features; their coenobia have large perforations between slender $\mathrm{H}$-shaped cells and their cell wall is smooth (not ornamented).

The other Pediastrum duplex-like strains form one well resolved lineage (BPP 1, ML 98), which is sister to a lineage composed of all $P$. boryanum-like strains. Both lineages form a strongly supported branch (BPP 1, ML 84).

The lineage composed of the other $P$. duplex-like strains is divided into two well resolved clades, the upper and the lower one.

In the upper clade (BPP 0.999, ML 79), a strongly 
supported GROUP II (Fig. 1; BPP 1, ML 99) is composed of 6 strains, mainly European $P$. duplex var. rugulosum (30.260911a, 14.280112 and 05.310112 from Poland, and ACOI 152 from Portugal), which have slender or robust $\mathrm{H}$-shaped cells and polygonal mesh with thickenings on the cell wall surface (Fig. 2a-f). The described group also contains one European strain identified as $P$. duplex var. asperum (ACOI 395 from the Czech Republic), which exhibits the same morphology as the strains identified to the variety rugulosum, and one Australian P. duplex strain (CR0501a), whose morphology (indistinctly perforated coenobia with slender $\mathrm{H}$-shaped cells covered with polygonal mesh and putative thickenings) is close to the morphology of the strains identified as the variety rugulosum.

In the upper clade, two European isolates of $P$. duplex var. duplex (ACOI 893 and 02.310811), whose coenobia possess a smooth cell wall surface (Fig. $2 \mathrm{~g}-\mathrm{i}$ ), also form a strongly supported monophyletic branch (GROUP IV in Fig. 1; BPP 0.986, ML 94). Similarly, three strains of Hydrodictyon spp. are gathered in a well supported branch in the upper clade composed mostly of $P$. duplex-like strains. However, the strains of $P$. duplex var. duplex are more closely related to the P. duplex var. rugulosum-like strains than to the strains of Hydrodictyon spp.

In the upper clade, three other strains are also located. One of them, identified as Pediastrum duplex var. cohaerens (ACOI 391 from Portugal; GROUP III in Fig. 1 ), exhibiting morphology typical of the $P$. duplex var. rugulosum strains from Poland, is most closely related to the strains of GROUP II (Fig. 1) representing this variety. However, the relationship is weakly supported $(\mathrm{BPP}<0.95, \mathrm{ML}<50)$. The second strain, identified as P. duplex (SL0401MN; GROUP V in Fig. 1) from North America, which possesses a cell wall surface ornamented as in the $P$. duplex var. rugulosum strains, is most closely related to the strains of GROUP IV (Fig. 1) representing the variety duplex, but again with weak support. The last strain in the upper clade represents $P$. duplex var. asperum (UTEX LB1364; GROUP VI in Fig. 1) from Europe, whose coenobia with robust $\mathrm{H}-$ shaped cells are covered with ridges. This strain is basal to a weakly supported branch $(\mathrm{BPP}<0.95, \mathrm{ML}<50)$ containing all the other strains of the upper clade.

The lower strongly supported clade (GROUP VII in Fig. 1; BPP 1, ML 100) contains three Australian Pediastrum duplex isolates (LB0503a, WR0401 and PL0501b), three P. angulosum strains (HL0201WI, ACOI 1354 and MB0403b) from distant localities (North America, Europe and Australia, respectively) and an Australian strain identified as Pseudopediastrum alternans? (LWiv0503b). The P. duplex strains have distinctly perforated coenobia with slender $\mathrm{H}$-shaped cells and cell wall ornamentation (polygonal mesh or wrinkles with clearly visible or questionable thickenings) resembling that of $P$. duplex var. rugulosum. The strain identified as Ps. alternans? has rounded U-shaped marginal cells which are typical of $P$. boryanum, but its cell wall ornamentation is composed of wrinkles without granules or thickenings. In GROUP VII, only the Australian Ps. alternans? strain and another strain from Australia identified as $P$. angulosum form a strongly supported branch (BPP 0.968, ML 85). The relationships between the other isolates of GROUP VII are weakly supported $(\mathrm{BPP}<0.95, \mathrm{ML}<70)$.

\section{Pediastrum boryanum-like strains}

The strains which represent morphology typical of, or close to, Pediastrum boryanum, except for the Australian Pseudopediastrum alternans? strain located in GROUP VII mentioned above and a European strain identified as Pediastrum sp. (RL0201FR; GROUP VIII in Fig. 1) forming small compact coenobia with long U-shaped marginal cells and cell walls covered with triangular mesh and medium dense granulation, are gathered in a strongly supported branch (BPP 0.996, ML 85). This branch is divided into two well resolved lineages (both having BPP 1 and ML 100).

One of these lineages (GROUP IX in Fig. 1) contains two strains, one identified as Pseudopediastrum brevicorne (ACOI 685, from Europe), and the second identified as Ps. cf. alternans (SAG 2440, from Africa). Whereas the morphology of the Ps. cf. alternans strain (Fig. $2 \mathrm{j}-1$ ) is well resolved (small compact coenobia with rounded or long U-shape cells covered with mesh and medium dense elongated granules), the typical morphology of the Ps. brevicorne strain is questionable as it often occurs in the form of planar or three-dimensional chaotic arrangements of cells (Fig. 3a-c).

This two-strained clade is sister to a lineage in which a strain identified as Ps. integrum (SAG 29.81, no locality) and a strain of Ps. kawraiskyi (SAG 35.81, from Europe) form a well supported clade (GROUP X in Fig. 1; BPP 1, ML 99). The coenobia of SAG 29.81 are small and compact, and their marginal cells are rounded O-shaped with one or two processes (Fig. 3d-e). Its cell wall ornamentation is composed of loosely arranged granules on a triangular or polygonal mesh (Fig. 3f).

The Pseudopediastrum integrum/ kawraiskyi clade (GROUP X in Fig. 1) is basal to all Ps. boryanum strains, single strains of Pediastrum cf. subgranulatum (19.300112), Ps. alternans (01.021213), Ps. brevicorne (SAG 261-7) and Ps. cf. integrum (11.310112), being gathered in a well supported (BPP 1, ML 100) branch. This branch, composed mostly of PS. boryanum strains, is divided into five more or less supported lineages.

The first lineage is weakly supported (BPP $<$ $0.95, \mathrm{ML}<50)$. It is divided into two branches. The first weakly supported branch is divided into three clades with two strains in each clade (GROUPS XI-XIII in Fig. 1). The first clade (GROUP XI; BPP 0.991, ML 81) is composed of two Polish lowland strains identified as $P S$. boryanum var. longicorne (04.020414 and 04.080210), sharing similar morphology, including the lack of perforations between cells, long U-shaped marginal cells 
and the type of wall ornamentation being a polygonal mesh with loosely situated rounded or distorted granules (Fig. 3g-1). The second clade (GROUP XII in Fig. 1; BPP 0.998, ML 79) is composed of morphologically very similar strains from Europe, including 02.290811a identified as Ps. boryanum var. longicorne (Fig. 4a-c) and ACOI 1466 identified as Ps. brevicorne f. granulatum (the synonym of Ps. boryanum var. longicorne). The strains have small or large coenobia, but share the lack of perforations, the long-U shape of marginal cells and loose granulation situated on a triangular or polygonal mesh. The rounded granules in 02.290811a are of different sizes (Fig. 4c). The third clade (GROUP XIII in Fig. 1; BPP 0.992, ML 50) is composed of two European strains differing in origin and morphology. The strain identified as Pediastrum cf. subgranulatum (19.300112) from a mountain lake in Poland has small distinctly perforated coenobia, robust $\mathrm{H}$-shaped marginal cells with short processes and loose granulation (Fig. 4d-f). In contrast, the strain identified as Pseudopediastrum boryanum var. forcipatum (SAG 87.81) from a lowland lake in Germany has small or large coenobia either lacking or with small perforations, long or rounded U-shape marginal cells, sometimes with converging processes, as well as dense or no granulation (Fig. $4 \mathrm{~g}-\mathrm{i}$ ).

The second branch of the first lineage is strongly supported (GROUP XIV in Fig. 1; BPP 0.996, ML 92). It includes a strain identified as Ps. cf. integrum (11.310112) and a strain identified as Ps. alternans (01.021213). Both originate from Polish water bodies, a mountain and a lowland lake, respectively. Although they both possess large coenobia, they clearly differ with respect to the presence of perforations between cells, the shape of marginal cells and the wall ornamentation (Figs $4 \mathrm{j}-1,5 \mathrm{a}-\mathrm{c}$ ).

The second lineage (GROUP XV in Fig. 1; BPP 1, ML 99) is composed of two European stains identified as Ps. boryanum var. boryanum (08.040414 and 02.310112; Fig. 5d-i), a North American strain of Ps. boryanum not identified to the variety level (IL0402MN) and a strain identified as PS. brevicorne (SAG 261-7, without locality information). The morphology of these strains is similar, but not identical (small or large compact coenobia with rounded or long U-shaped marginal cells and triangular mesh with medium dense granules on the cell wall surface, not readily visible in the strain IL0402MN). In this lineage, the strain of Ps. boryanum var. boryanum no. 08.040414 is more closely related to the strain SAG 261-7 of Ps. brevicorne than to the other strain of PS. boryanum var. boryanum (02.310112).

The third lineage (GROUP XVI in Fig. 1; BPP 0.979, ML 94) is composed of three strains identified as Ps. boryanum var. cornutum (19.090508, UTEX LB470 and ACOI 1009, from European lowland localities). Among them, the morphology is resolved for 19.090508 (Fig. 5j-1) and UTEX LB470. Their small coenobia with small perforations have a cell wall surface covered with mesh and granules, but their marginal cells are rounded
$\mathrm{U}-$ or robust $\mathrm{H}-$ shaped, respectively. The fourth lineage (GROUP XVII in Fig. 1; BPP 1, ML 98) is composed of two morphologically very similar strains from Europe and North America, including one identified as Ps. boryanum var. boryanum (ACOI 75; Fig. 6a-c) and the second not identified to the variety level (EL0203CT). The strains have small compact coenobia, rounded U-shaped marginal cells and loose granules on the cell wall surface. The granules in ACOI 75 are small and delicate (Fig. 6c). The last lineage (GROUP XVIII in Fig. 1) contains only one European strain identified as PS. boryanum cf. var. forcipatum (07.030414). It has small compact coenobia, rounded U-shape marginal cells and dense granulation and a triangular mesh on the cell wall surface (Fig. 6d-f).

\section{DisCUSSION}

In the present study, the phylogenetic relationships among some varieties of Pediastrum boryanum and P. duplex s.l., morphologically similar taxa and other representatives of the family Hydrodictyaceae, shown on the basis of a joint analysis of two molecular markers, including 26S rDNA and chloroplast $r b c \mathrm{~L}$, were resolved.

All strains identified as $P$. duplex or its varieties, including duplex, asperum (A. Braun) Hansgirg, cohaerens Bohlin, gracillimum W. West et G.S. West and rugulosum Raciborski, form seven different groups in the obtained phylogenetic tree. Similarly, in the studies by McManus \& Lewis (2011), Jena et al. (2014) and LenARCZYK \& McManus (2016), P. duplex strains were divided in two or three groups, including strains identified as Lacunastrum gracillimum (W. West et G.S. West) H. McManus (formerly P. duplex var. gracillimum) and forming a monophyletic clade which was most closely related to Monactinus Corda strains. The latter taxon, formerly known as P. simplex Meyen, forms coenobia composed of triangular marginal cells with only one lobe and process, unlike $P$. boryanum and $P$. duplex s.1. (KOMÁREK \& JANKOVSKÁ 2001). The present results show the monophyly of L. gracillimum (GROUP I) on the basis of strains from distant localities in Europe and North America, confirming the results of a number of previous authors (McManus \& Lewis 2011; MCMANUS et al. 2011; JENA et al. 2014).

Besides the variety gracillimum, having slender $\mathrm{H}$-shaped cells and a smooth cell wall surface, KOMÁREK \& JANKOVSKÁ (2001) distinguished three other varieties in $P$. duplex, having robust $\mathrm{H}-$-shaped cells and various types of cell wall surface. The surface can be smooth in the variety duplex or ornamented in the varieties asperum (araneose ornamentation composed of coarse ridges) and rugulosum (net-like ornamentation composed of fine wrinkles and thickenings called verrucae). One of the strains analyzed in this study, ACOI 391 from Europe (GROUP III), was named P. duplex var. cohaerens in the literature. KOMÁREK \& JANKOVSKÁ (2001) treated this 


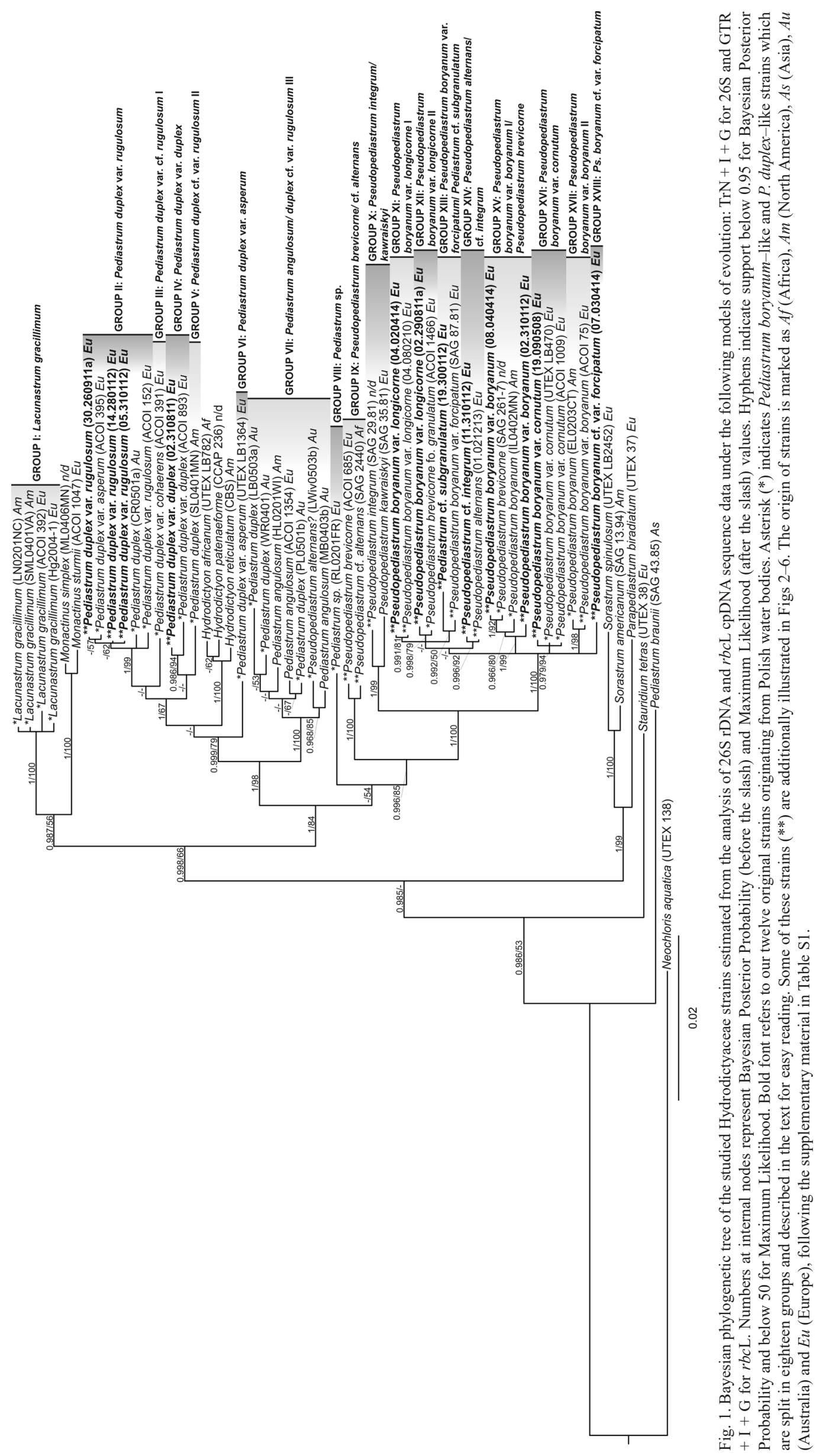



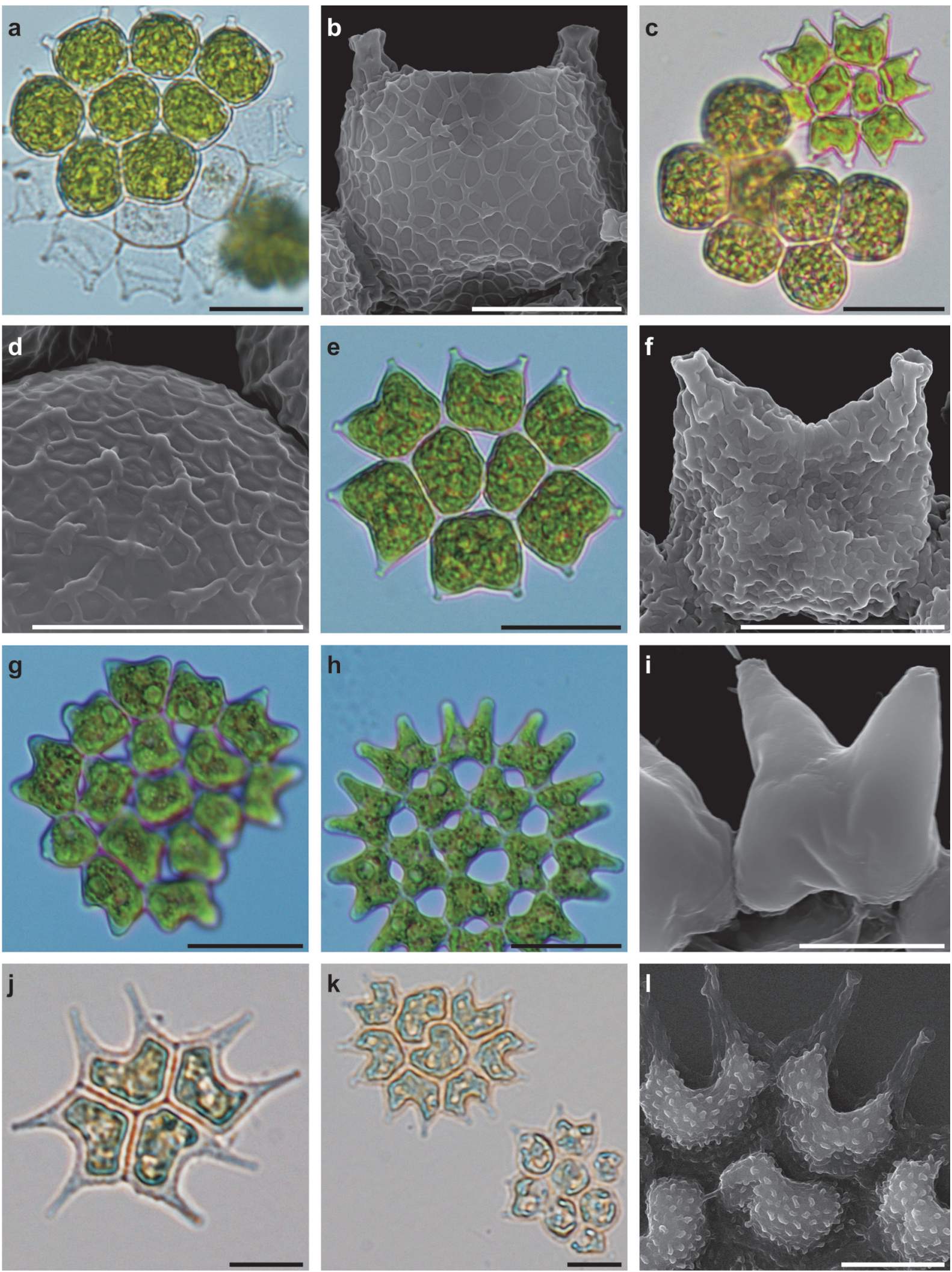

Fig. 2. Pediastrum s.l. strains analyzed morphologically using light and scanning electron microscopy. (a-b) $P$. duplex var. rugulosum (30.260911a); (c-d) P. duplex var. rugulosum (14.280112); (e-f) P. duplex var. rugulosum (05.310112); (g-i) P. duplex var. duplex (02.310811); (j-1) Pseudopediastrum cf. alternans (SAG 2440); (a, c, e, g-h, j-k) scale bars $20 \mu \mathrm{m}$; (b, d, f, i \& 1) scale bars $5 \mu \mathrm{m}$. 

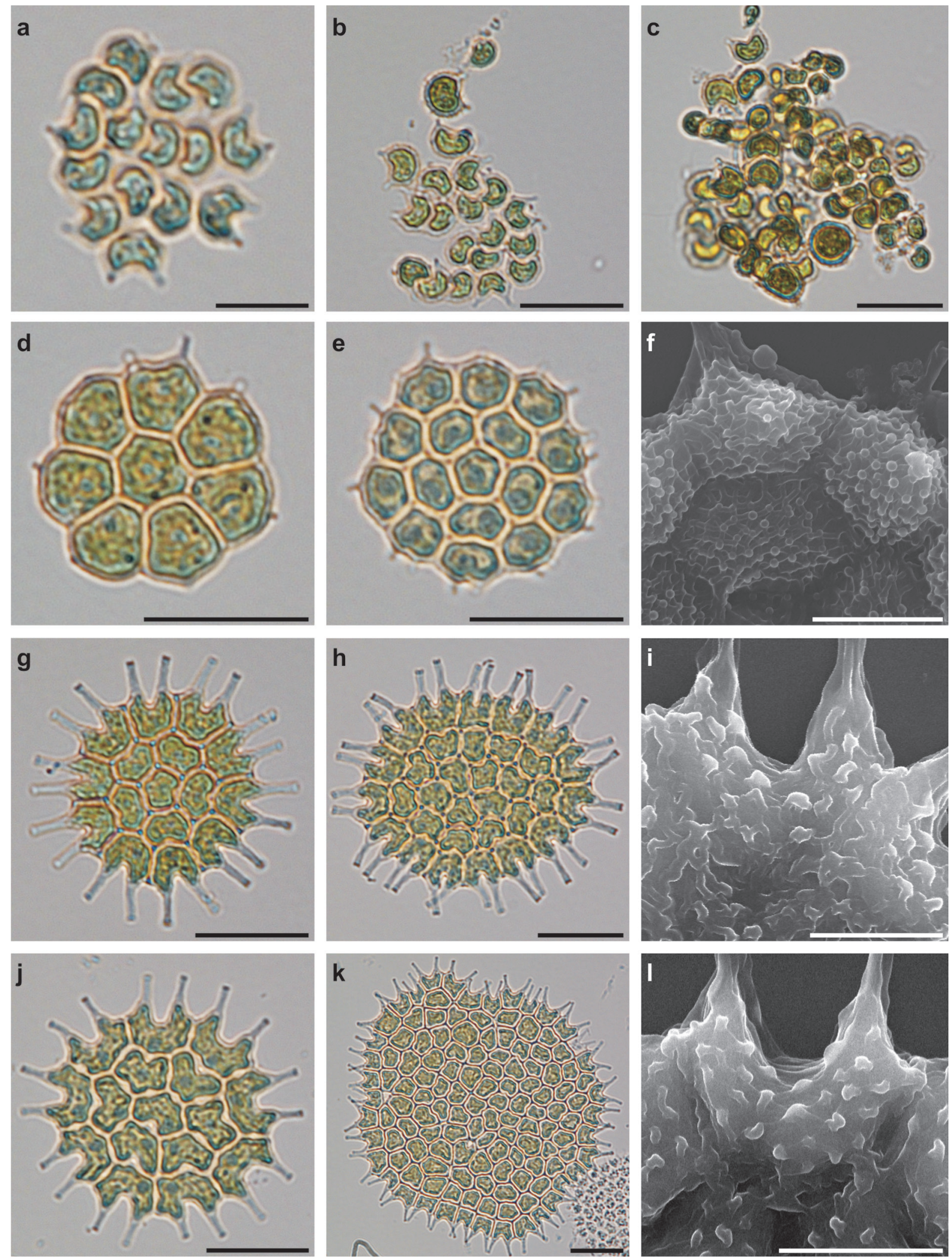

Fig. 3. Pediastrum s.l. strains analyzed morphologically using light and scanning electron microscopy. (a-c) Pseudopediastrum brevicorne (ACOI 685); (d-f) Ps. integrum (SAG 29.81); (g-i) Ps. boryanum var. longicorne (04.020414); (j-1) Ps. boryanum var. longicorne (04.080210); (a-b, d-e, g-h, j-k) scale bars $20 \mu \mathrm{m}$; (c) scale bar $10 \mu \mathrm{m}$; (f, i \& 1) scale bars $5 \mu \mathrm{m}$. 

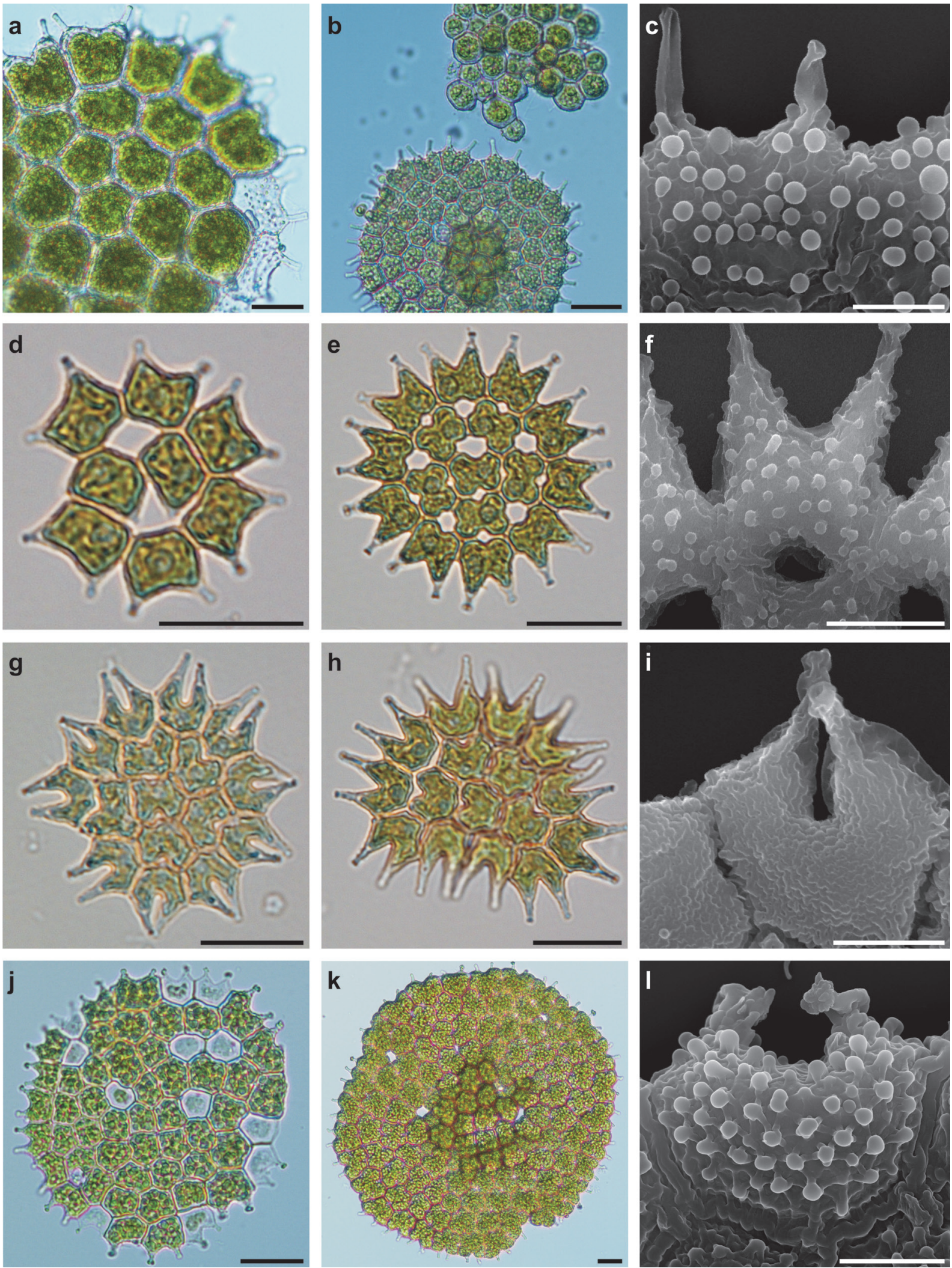

Fig. 4. Pediastrum s.l. strains analyzed morphologically using light and scanning electron microscopy. (a-c) Pseudopediastrum boryanum var. longicorne (02.290811a); (d-f) P. cf. subgranulatum (19.300112); (g-i) Ps. boryanum var. forcipatum (SAG 87.81); (j-1) Ps. cf. integrum (11.310112); (a-b, d-e, g- h, j-k) scale bars $20 \mu \mathrm{m} ;(\mathrm{c}, \mathrm{f}, \mathrm{i} \& \mathrm{l})$ scale bars $5 \mu \mathrm{m}$. 

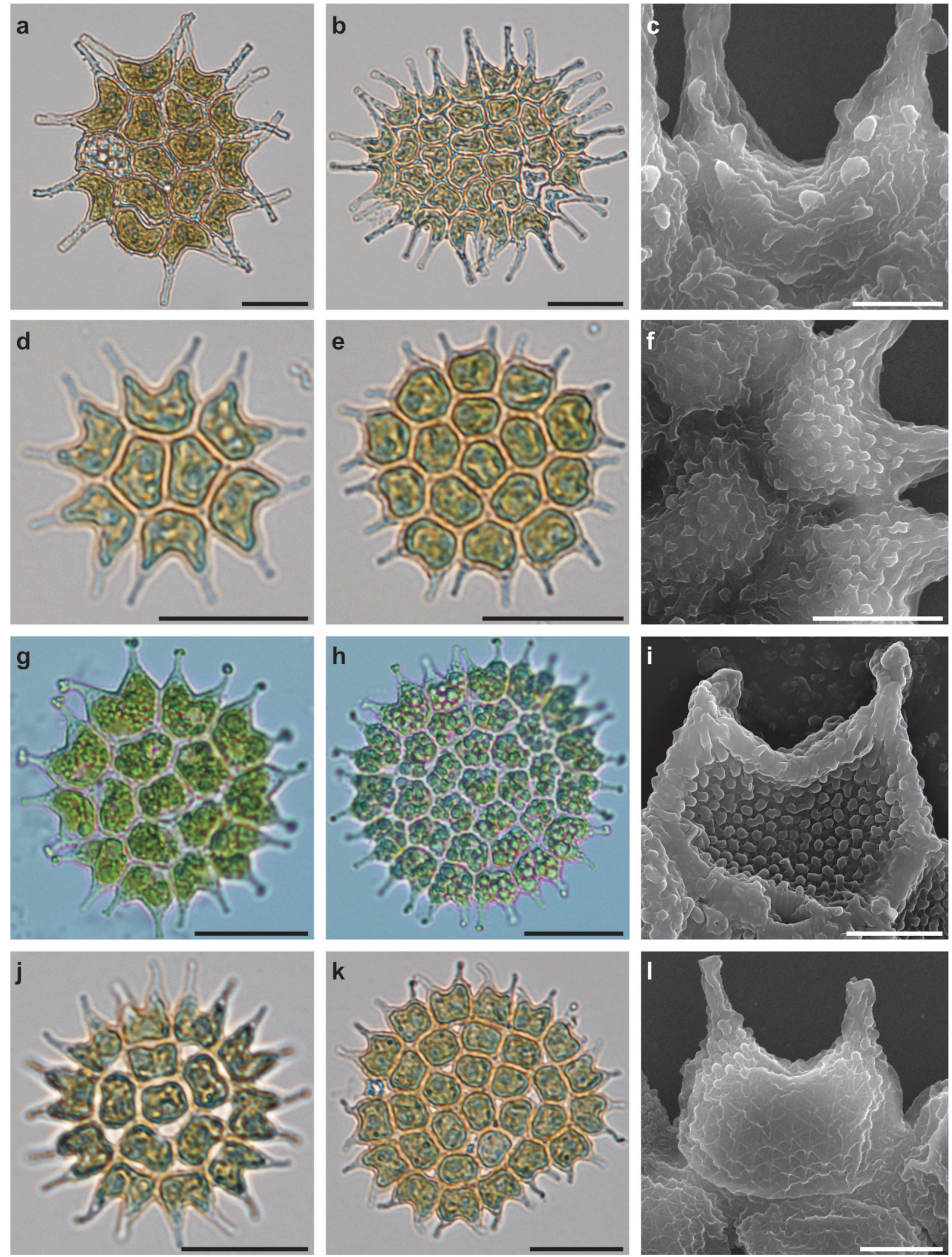

Fig. 5. Pediastrum s.l. strains analyzed morphologically using light and scanning electron microscopy. (a-c) Pseudopediastrum alternans (01.021213); (d-f) Ps. boryanum var. boryanum (08.040414); (g-i) Ps. boryanum var. boryanum (02.310112); (j-1) Ps. boryanum var. cornutum (19.090508); (a-b, d-e, g- h, j-k) scale bars $20 \mu \mathrm{m}$; (c, f, i \& 1) scale bars $5 \mu \mathrm{m}$. 

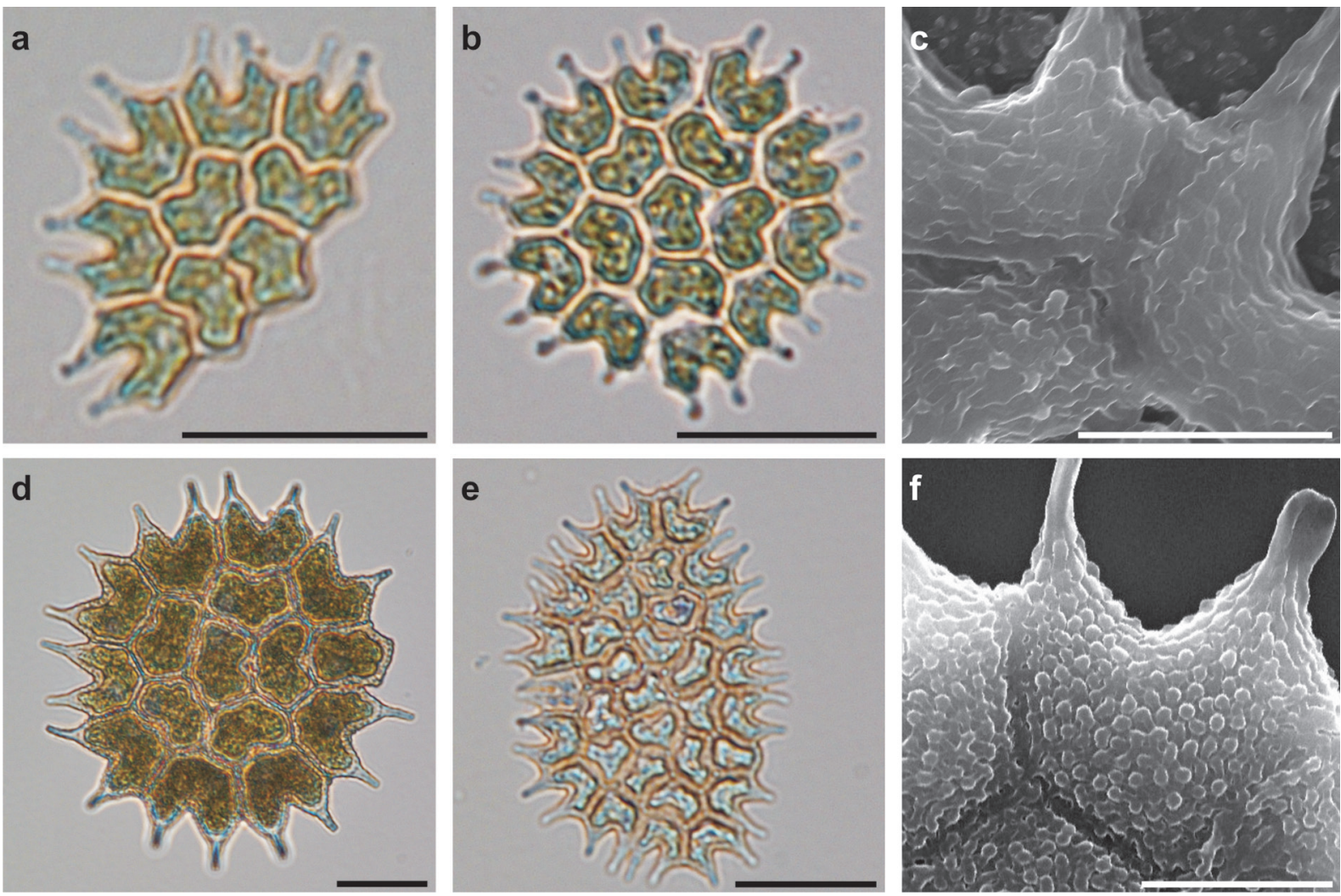

Fig. 6. Pediastrum s.l. strains analyzed morphologically using light and scanning electron microscopy. (a-c) Pseudopediastrum boryanum var. boryanum (ACOI 75); (d-f) Ps. boryanum cf. var. forcipatum (07.030414); (a-b, d-e) scale bars $20 \mu \mathrm{m}$; (c \& f) scale bars $5 \mu \mathrm{m}$.

variety as a synonym of $P$. argentinense Bourrelly et Tell. However, the morphology of this strain corresponds more to that of other $P$. duplex var. rugulosum strains analyzed in this study than to the morphology of $P$. argentinense (Table 1). Morphological differences between P. duplex var. rugulosum and $P$. argentinense were previously analyzed in detail by LENARCZYK et al. (2015).

In the present study, two strains identified as P. duplex var. duplex from Europe formed a well supported lineage (GROUP IV). In the study by McManUs \& LEWIS (2011), one of these strains (ACOI 893) with a smooth wall surface (as mentioned by McManus \& LEwIs 2011) was closely related to a strain identified as P. duplex having also a smooth wall surface (SR0201NJ) and representing the morphotype of the variety duplex, as well as another strain identified as $P$. duplex, but without information on the cell shape and wall surface (SAG 84.80). If the latter strain also possesses a smooth wall surface, it will make it more plausible that $P$. duplex var. duplex is a monophyletic taxon.

Four European (Polish, Portuguese) strains identified as $P$. duplex var. rugulosum, as well as one European (Portuguese) strain with the given name P. duplex var. asperum (ACOI 395) and one Australian strain identified as P. duplex (CR0501a), which exhibit the same or similar morphology to $P$. duplex var. rugulosum, formed a well supported clade (GROUP II) in the obtained phylogenetic tree. The strains should be regarded as true representatives of $P$. duplex var. rugulosum, as their morphology generally corresponds to the original description of the taxon by RACIBORSKI (1889) from its European locus classicus (Poland, Chełmek). Small differences in the wall ornamentation between the European strains (polygonal mesh and thickenings), the Australian strain (polygonal mesh and putative thickenings), the description by KoMÁREK \& JANKOVSKÁ (2001) (net-like ornamentation composed of fine wrinkles with thickenings called verrucae) and the original description (membrana rugosa, undulato verruculosa, granulationes dense dispositae, inaequales, irregulares, nonnunquam paullo elongatae) seem to be connected with different spatial resolution of available microscopes and ontogenetic variability (the change from a mesh to wrinkles as a result of wall stretching) observed by PARRA (1979). Similarly, differences in the $\mathrm{H}$-shaped marginal cells (robust or slender) resulting in small or large perforations in coenobia of the strains in GROUP II can result from culture conditions, as observed for $P$. duplex by Neustupa \& HODAČ (2005), and should not be treated as diagnostic characters.

The other strains of $P$. duplex from distant localities (Australia and North America) with cell wall ornamentation typical of, or close to, the variety rugulosum (polygonal mesh with thickenings or wrinkles with putative thickenings) are placed in three separate lineages (GROUPS III, V and VII), which seem to represent at least three new taxa. Similarly, $P$. duplex strains possessing wavy and netlike ornamentation patterns were grouped in several separate lineages in the phylogenetic analysis 
made by McMANus \& Lewis (2011). One of the present lineages (GROUP VII) corresponds to Group II in the tree presented by McMANus \& LEwIS (2011). Both groups contain not only strains identified as $P$. duplex, but also representatives of Australian, European and North American $P$. angulosum exhibiting morphology different from $P$. boryanum and $P$. duplex, as well as an Australian strain identified as Pseudopediastrum alternans? (LWiv0503b) with U-shaped cells, resembling that of $P$. boryanum. However, detailed morphological analysis, e.g. with the help of geometric morphometric methods previously used for taxonomical delimitation of another P. duplex-like morphotype, L. gracillimum (McManus et al. 2011), are needed to make sound conclusions on the systematics of the $P$. duplex var. rugulosum-like morphotypes. MCMANUS \& LEWIS (2011) stated that not only other forms of morphological information, but also more variable molecular markers are needed to clarify the relationships between $P$. duplex var. rugulosum-like, P. angulosum and Ps. alternans-like morphotypes.

Only one strain of $P$. duplex var. asperum (UTEX LB1364) from a European locality, except for the misidentified ACOI 395, has been molecularly analysed so far (MCMANUS \& LEWIS 2005, 2011; LENARCZYK \& MCMANUs 2016). It forms a separate lineage (GROUP VI) in the present study. However, it is difficult to infer the phylogenetic position of $P$. duplex var. asperum from only one strain. In previous studies, the strain has been mostly related to a strain of Pediastrum sp. (MB0404) from Australia (McMANus \& LEWIS 2011) or to several P. duplex, Pediastrum sp. and P. angulosum (Ehrenberg) ex Meneghini strains (McManus \& LeWIS 2005; LenARCZYK \& McManus 2016). The cell wall ornamentation of $P$. angulosum is also araneose (so far observed only in $P$. duplex var. asperum and $P$. angulosum), but the cell shape is different to that of $P$. duplex var. asperum. More strains representing the $P$. duplex var. asperum morphotype are needed to determine the phylogenetic position of the taxon and its taxonomical homogeneity.

The present study confirms the polyphyletic nature of $P$. boryanum s.1. MCMANUS \& Lewis (2005) stated that the species is monophyletic, but sequencing a higher number of strains showed its paraphyletic character (MCMANUS \& LEWIS 2011; JENA et al. 2014; LENARCZYK \& MCMANUS 2016).

Altogether, five varieties of $P$. boryanum s.l. were subjected to the present phylogenetic analysis, including boryanum (as Pseudopediastrum boryanum (Turpin) E. Hegewald var. boryanum), brevicorne A. Braun (as Ps. brevicorne (A. Braun) Jena et C. Bock), cornutum Sulek (as Ps. boryanum var. cornutum), forcipatum Parra (as Ps. boryanum var. forcipatum) and longicorne Reinsch (as Ps. boryanum var. longicorne and Ps. brevicorne f. granulatum (Raciborski) Parra).

Only the variety cornutum formed a well supported monophyletic clade composed of more strains (GROUP XVI). LENARCZYK (2014) and LENARCZYK \&
WolowsKi (2016) found that the coenobia of $P$. boryanum var. cornutum and $P$. boryanum var. perforatum (Raciborski) Nitardy are very similar in cell shape and wall ornamentation. Similarly, NIELSEN (2000) questioned the distinction between these varieties. However, no strains of the variety perforatum have been included in molecular studies to date, so the taxonomical problem remains unresolved.

Only one strain representing Ps. boryanum var. forcipatum was included in the present phylogenetic analysis. A morphologically similar strain, identified as Ps. boryanum cf. var. forcipatum, slightly differed in the marginal cell shape (processes not converging). These two strains did not form a common lineage (GROUPS XIII and XVIII) in the present study, suggesting that the latter strain represents a new taxon, but more strains with such morphology are needed to confirm its taxonomic position.

Pediastrum boryanum var. forcipatum is also morphologically similar to $P$. boryanum var. pseudoglabrum PARRA. These two taxa generally differ in cell wall ultrastructure, but some intermediate forms were also observed by LENARCZYK (2014) and LENARCZYK \& Wolowski (2016). Thus, it was suggested that the variety pseudoglabrum, usually forming a scabrate wall pattern (composed of tiny verrucae and punctae), can be a form of the variety forcipatum, usually forming a granulate wall pattern (composed of granules situated densely in regular rows, and punctae), but with wall ornamentation not well developed. No strains representing the variety pseudoglabrum have been molecularly analysed so far, so the relationships between these two taxa remain unclear.

The only strain representing Ps. boryanum var. forcipatum was closely related to a strain resembling Pediastrum subgranulatum, which exhibits a very different morphology (GROUP XIII). The marginal cells of the $P$. cf. subgranulatum strain were a little longer than presented in the pictures of P. subgranulatum in the monograph by KoMÁREK \& JANKOvsKá (2001), who elevated $P$. duplex var. subgranulatum RACIBORSKI to species rank as a new combination. Pediastrum subgranulatum combines features typical of $P$. boryanum, namely a reticulate wall pattern with regularly disposed granules, as well as including perforated coenobia, $\mathrm{H}$-shaped cells and very short processes (rosettes), typical of $P$. duplex. Previously, $P$. subgranulatum had been classified as a variety of $P$. duplex, with the basionym $P$. duplex var. subgranulatum Raciborski, and included the synonyms P. duplex var. typicum f. punctatum Krieger or P. duplex var. punctatum Parra (KoMÁREK \& JANKOVsKÁ 2001). In both $P$ s. boryanum var. forcipatum and $P$. cf. subgranulatum large evolutionary changes have occurred in this lineage, which can be explained by adding more strains representing both the Ps. boryanum var. forcipatum - and P. subgranulatum-like morphologies.

Except for Ps. boryanum var. cornutum and Ps. boryanum var. forcipatum, the varieties boryanum, 
brevicorne and longicorne, proved to be complex taxa as strains of each of the varieties were dispersed in different clades.

The strains representing the variety boryanum are to be found in two separate lineages (GROUPS XV and XVII). The lineages differ in the cell wall granulation, which is of medium density in GROUP XV (not visible in IL0402MN) and loose in GROUP XVII. High variability in P. boryanum var. boryanum morphology, including cell shape and granule density, was previously observed by LENARCZYK (2014) and LENARCZYK \& WolowsKi (2016).

In the present study, two strains identified as PS. brevicorne (formerly Pediastrum boryanum var. brevicorne) are grouped in distant lineages with different species, with $P$. boryanum var. boryanum and $P$. boryanum (GROUP XV), and with Ps. cf. alternans (GROUP IX). McMANUS \& LEWIS (2011) similarly found two strains identified as Ps. boryanum var. brevicorne in two separate lineages, with Ps. boryanum and Pediastrum sp., further confirming the polyphyletic nature of the variety. Based only on morphological studies, NIELSEN (2000) stated that the variety brevicorne should be rejected and subsumed into the nominate variety. Molecular studies indicate that the taxon should be divided into two other taxa, maybe one of them representing the variety boryanum. However, additional detailed morphometric analyses are required. The variety longicorne was divided into two clades (GROUPS XI and XII). Similarly, LENARCZYK \& SALUGA (2013) observed a division of the variety longicorne into lowland and mountainous groups of Polish strains in a phylogenetic tree based on $18 \mathrm{~S}, 26 \mathrm{~S}$ and ITS rDNA sequences. In the present study, the cell wall surface was shown to be covered with prominently rounded granules in the Polish mountainous strain from GROUP XII, but with more or less rounded granules in the Portuguese strain from the same group and the Polish lowland strains from GROUP XI. The morphological differences shown in Polish strains were pointed out by LENARCZYK \& WoŁowsKi (2016), who also suggested the complexity of Ps. boryanum var. longicorne. REINSCH (1867), who described the variety longicorne, mentioned that its cell wall surface is smooth. It could have been connected with low spatial resolution of available microscopes, as subsequent researchers (e.g. PARra 1979; KomÁreK \& JANKOVSKÁ 2001; LENARCZYK \& WolOWSKI 2016) have always observed ornamented cell walls covered with rarely disposed granules.

One Polish strain (01.021213) identified as PS. alternans in the present study has coenobium and cell shape typical of this species, described by NYGAARD (1949) as Pediastrum alternans from Danish lakes (LENARCZYK \& McManus 2016). KowAlsKa \& WolowsKi (2010) suggested that the presence of verrucae, not observed by NyGAARD (1949), but observed on the cell wall surface of Ps. alternans found in the Polish coastal lakes (and on the surface of the 01.021213 strain) indicates a probable new variety. The 01.021213 strain was located within a clade dominated with Ps. boryanum strains. The close relationship of Ps. alternans and Ps. boryanum was previously stated also by LENARCZYK \& MCMANUs (2016). In the present study, the 01.021213 strain (PS. alternans) was most closely related (GROUP XIV) to a strain resembling Ps. integrum (11.310112), which exhibited different morphology, i.e., loose cell wall granulation. This is the case of closely related, but morphologically different taxa, similar to that of Ps. boryanum var. forcipatum and $P$. cf. subgranulatum, where adding more strains representing their morphology could clarify large evolutionary changes occurring in the lineage. The 11.310112 strain identified as Ps. cf. integrum may represent a new species, but detailed morphological analyses are necessary to prove its distinctiveness.

Detailed morphological analyses, using geometric morphometric methods, which allow analyzing shape as a whole without reducing it to single measurements, like length or width (ZELDITCH et al. 2004), along with molecular analyses, revealed that the African strain SAG 2440, previously identified as Ps. alternans by JENA et al. (2014), is not a representative of this species (LenARCZYK \& McManus 2016). Thus, it was identified as PS. cf. alternans in the present study. In both the present and previous studies (LENARCZYK \& MCMANUS 2016), the strain forms a separate lineage (GROUP IX) with the strain ACOI 685, identified as Ps. brevicorne or PS. boryanum var. brevicorne, respectively. Limited data on appearance of the latter strain do not allow us to make any definite conclusions regarding typical morphology in this lineage.

Pseudopediastrum integrum (formerly Pediastrum integrum) formed a clade (GROUP X) with Ps. kawraiskyi which exhibits different morphology to that of Pediastrum boryanum and P. duplex. The clade was sister to a clade composed mostly of Ps. boryanum strains. Similar results were obtained by MCMANUS \& LEWIS (2005, 2011), JENA et al. (2014) and LENARCZYK \& MCManus (2016).

The RL0201FR strain identified as Pediastrum sp. was the last strain representing $P$. boryanum-like morphology. In the present study, it was placed close to Pseudopediastrum taxa, although the relationship was weakly supported (GROUP VIII). In the study by MCMANus \& LEWIS (2011), the strain was grouped together with Pseudopediastrum strains based on $r b c \mathrm{~L}$ data or closer to Pediastrum strains based on 26S rDNA data. According to MCMANUS \& LEWIS (2011), the RL0201FR strain forms a common lineage with a morphologically similar strain, SAG 2062, which was named P. berolinense by BucHHEIM et al. (2005).

In the case of two strains, SAG 2440 (Pseudopediastrum cf. alternans) and SAG 87.81 (Ps. boryanum var. forcipatum), there were small differences in their morphology observed by the present authors and shown in the literature (Table 1). The variation could have resulted from differences in culture media and culture conditions. So far, only a few experimental studies on the impact 
of environmental factors on morphological variability of Pediastrum s.l. have been conducted (e.g., NEUSTUPA \& HodaČ 2005; RoJo et al. 2007; LENARCZYK 2016; LENARCZYK \& MCMANUS 2016), and they need to be continued to make taxonomic conclusions more reliable.

\section{Conclusions}

The gene topologies reveal many discrepancies in the morphological features used to delimit analysed taxa. Adding more strains representing the morphology of Pediastrum duplex, as well as representing both the analysed varieties of Pseudopediastrum boryanum and other varieties of the species, including vars. campanulatum WILle, caribeanum Comas, pseudoglabrum Parra and perforatum (Raciborski) Nitardy, is necessary to clarify the relationships in these very diverse groups. Even though two molecular markers with long sequences were used in the present study, the distances between strains in the Pseudopediastrum clade were very short. Thus, it is important to add information from more variable molecular markers. In the case of such variable taxa with intricate cell shape as observed in $P$. boryanum, $P$. duplex s.l. and similar species, detailed morphological analyses using modern methods like geometric morphometrics are required. The present study shows that taxonomical changes within both analysed species need to be made.

\section{ACKNOWLEDGEMENTS}

The authors are especially indebted to Michał Ronikier for his valuable suggestions on the phylogenetic analyses. They are also grateful to Rod D. Seppelt for his help in English correction. They thank Jolanta Piatek and Anna Łatkiewicz for their assistance during SEM observations. This work was financed by the statutory fund of the W. Szafer Institute of Botany of the Polish Academy of Sciences, including an in-house grant for young scientists in 2012.

\section{REFERENCES}

Buchheim, M.A.; Buchheim, J.A.; Carlson, T.; Braband, A.; Hepperle, D.; Krienitz, L.; Wolf, M. \& Hegewald, E. (2005): Phylogeny of the Hydrodictyaceae (Chlorophyceae): inferences from rDNA data. - J. Phycol. 41: 1039-1054.

Chang, T.-P. \& Chang-Schneider, H. (1980): Zur Variabilität von Pediastrum boryanum (Turpin) Meneghini. Algological Studies 26: 53-62.

Darriba, D.; TABOAda, G.L.; Doallo, R. \& Posada, D. (2012): jModelTest 2: more models, new heuristics and parallel computing. - Nature Methods 9: 772.

Gouy, M.; Guindon, S. \& Gascuel, O. (2010): SeaView version 4: a multiplatform graphical user interface for sequence alignment and phylogenetic tree building. - Mol. Biol. Evol. 27: 221-224.

HALL, T.A. (1999): BioEdit: a user-friendly biological sequence alignment editor and analysis program for Windows 95/98/NT. - Nuc. Acid. S. 41: 95-98.

Jena, M.; Bock, C.; Behera, C.; Siba, P.; Adhikary, S.P. \&
KRIENITZ, L. (2014): Strain survey on three continents confirms the polyphyly of the genus Pediastrum (Hydrodictyaceae, Chlorophyceae). - Fottea 14: 63-76.

KomÁreK, J. \& Fotт, B. (1983): Chlorophyceae (Grünalgen), Ordnung: Chlorococcales. - In: Huber-Pestalozzi, G. (ed.): Das Phytoplankton des Süßwassers, Teil 7, Hälfte 1. -1044 pp., E. Schweizerbart'sche Varlagsbuchhandlung (Nägele u. Obermiller), Stuttgart.

KomÁReK, J. \& JANKovskÁ, V. (2001): Review of the green algal genus Pediastrum: implication for pollen-analytical research. - Bibl. Phycol. 108: 1-127.

KowAlsKa, J. \& Wolowski, K. (2010): Rare Pediastrum species (Chlorophyceae) from Polish coastal lakes. - Acta Soc. Bot. Pol. 79: 225-233.

LenarcZYK, J. (2014): The algal genus Pediastrum Meyen (Chlorophyta) in Poland. - 104 pp., Institute of Botany PAS, Kraków.

LenarczyK, J. (2015): Pediastrum Meyen sensu lato (Chlorophyceae) in the phytoplankton of lowland and upland water bodies of Central Europe (Poland). - Fottea 15: 165-177.

LENARCZYK, J. (2016): Morphological plasticity of the microscopic green alga Pseudopediastrum boryanum (Chlorophyceae) under varying nutrient concentration. - Nova Hedwigia 102: 373-390.

LenarczyK, J.; Kolaczek, P.; Jankovská, V.; Turner, F.; KarpińsKa-KolaczeK, M.; Pini, R.; PęDZiszewsKa, A.; Zimny, M.; STIVRins, N. \& SZymCZYK, A. (2015): Palaeoecological implications of the subfossil Pediastrum argentinense-type in Europe. - Review of Palaeobotany and Palynology 222: 129-138.

LenarCZyK, J. \& McManus, H.A. (2016): Testing the boundaries of the green algal species Pediastrum alternans (Chlorophyceae) using conventional, geometric morphometric and phylogenetic methods. - Phycologia 55: 515-530.

LenarczyK, J. \& Saluga, M. (2013): What do classical taxonomy and molecular data reveal about diversity of Pediastrum taxa in the Western Carpathians? Preliminary results. - Acta Botanica Cracoviensia. Series Botanica 55, suppl. 1: 54.

LeNARCZYK, J. \& Wolowski, K. (2016): Phenotypic plasticity of wall ultrastructure in the green alga Pediastrum s.l. (Chlorophyta, Sphaeropleales). - Pol. Bot. J. 61: 73-88.

McManus, H. \& Lewis, L.A. (2005): Molecular phylogenetics, morphological variation and colony-form evolution in the family Hydrodictyaceae (Sphaeropleales, Chlorophyta). - Phycologia 44: 582-595.

McManus, H. \& Lewis, L.A. (2011): Molecular phylogenetic relationships in the freshwater family Hydrodictyaceae (Sphaeropleales, Chlorophyceae), with an emphasis on Pediastrum duplex. - J. Phycol. 47: 152-163.

McManus, H.; Lewis, L.A. \& Schultz, E.T. (2011): Distinguishing multiple lineages of Pediastrum duplex with morphometrics and a proposal for Lacunastrum gen. nov. - J. Phycol. 47: 123-130.

Meneghini, J. (1840): Synopsis Desmidiearum hucusque cognitarum. - Linnaea 14: 201-240.

Meyen, F.J.F. (1829): Beobachtungen über einige niedrige Algenformen. - Nova Acta Phys.-Med. Acad. Caes. Leop.-Carol. Nat. Cur. 14: 771-778.

NäGELI, C. (1849): Gattungen einzelliger Algen. - 139 pp., Friedrich Schulthess, Zürich.

Neustupa, J. \& Hodač, L. (2005): Changes in shape of the coenobial cells of an experimental strain of Pediastrum 
duplex var. duplex (Chlorophyta) reared at different pH. - Preslia 77: 439-452.

Nguyen, H. D.; Jančič, S.; MeIJer, M.; TANney, J. B.; Zalar, P.; Gunde-Cimerman, N. \& Seifert, K. A. (2015): Application of the phylogenetic species concept to Wallemia sebi from house dust and indoor air revealed by multi-locus genealogical concordance. - PLoS One 10: $\mathrm{e} 0120894$.

NiELSEN, H. (2000): Morphometric analysis of cell wall sculpture in seven infraspecific taxa of Pediastrum boryanum (Sphaeropleales, Chlorophyta) and its taxonomic implications. - Phycologia 39: 36-49.

NygaARD, G. (1949): Hydrobiological studies on some Danish ponds and lakes. Part II: The quotient hypothesis and some new or little known phytoplankton organisms. - Kongel. Danske Vidensk. Selsk. Biol. Skr. 7: 1-293.

Parra, O.O. (1979): Revision der Gattung Pediastrum Meyen (Chlorophyta). - Bibl. Phycol. 48: 1-186.

RACIBORSKI, M. (1889): Przegląd gatunków rodzaju Pediastrum. - Rozpr. i Spr. Wydz. Mat.-Przyr. A.U. 20: 1-37.

ReINSCH, P. (1867): Die Algenflora des mittleren Theiles von Franken. - 238 pp., Verlag von Wilhelm Schmid, Nürnberg.

Rojo, C.; Segura, M.; Rodrigo, M.A. \& Salazar, G. (2007): Factors controlling the colonial structure of Pediastrum tetras (Chlorophyceae). - Hydrobiologia 617: 143-155.

Ronquist, F.; Teslenko, M.; Van Der Mark, P.; Ayres, D.L.; Darling, A.; Höhna, S.; Larget, B.; LiU, L.; Suchard, M.A. \& HuelsenBeCK, J.P. (2012): MrBayes 3.2: efficient Bayesian phylogenetic inference and model choice across a large model space. - Systematic Biology 61: 539-542.

SCHWARZ, G. (1978): Estimating the dimension of a model. - The Annals of Statistics 6: 461-464.

Stamatakis, A. (2006): RAxML-VI-HPC: maximum likelihood-based phylogenetic analyses with thousands of taxa and mixed models. - Bioinformatics 22: 2688-2690.

Stamatakis, A.; Hoover, P. \& Rougemont, J. (2008): A rapid bootstrap algorithm for the RAxML web servers. Systematic Biology 57: 758-771.
StAMATAKIs, A. (2014): RAxML version 8: a tool for phylogenetic analysis and post-analysis of large phylogenies. - Bioinformatics 30: 1312-1313.

SuleK, J. (1969): Taxonomische Übersicht der Gattung Pediastrum Meyen. - In: FotT, B. (ed.): Studies in Phycology. - pp. 197-261, Publishing House of the Czechoslovak Academy of Sciences, Praque.

SutKowy, M. (2017): Charakterystyka wzrostu biomasy zielenicy Pseudopediastrum boryanum w hodowli fotobioreaktorowej oraz możliwość jej wykorzystania do biosorpcji jonów $\mathrm{Cr}(\mathrm{VI})$ [PhD thesis]. - 110 pp., Uniwersytet Kazimierza Wielkiego, Wydział Nauk Przyrodniczych, Instytut Biologii Eksperymentalnej, Zakład Biotechnologii, Bydgoszcz.

TuRPIN, P.J.F. (1828): De la description de plusieurs genres et espèces nouvelles très remarquables, découverte parmi les productions végétales et microscopiques. - Memoir. Mus. Natl. Hist. 16: 295-344.

Zelditch, M.L.; Swiderski, D.L.; Sheets, H.D. \& FinK, W.L. (2004): Geometric morphometrics for biologists: a primer. - 443 pp., Elsevier Academic Press, New York.

Supplementary material

the following supplementary material is available for this article:

Table S1. Origin and the GenBank accession numbers of the studied Pediastrum s.1. strains and the outgroup Neochloris aquatica STARR (UTEX 138).

This material is available as part of the online article (http:/ fottea.czechphycology.cz/contents)

(C) Czech Phycological Society (2018)

Received October 17, 2017

Accepted April 4, 2018 